\title{
Moränen versus Till: Empfehlungen für die Beschreibung, Interpretation und Klassifikation glazialer Landformen und
} Sedimente

\author{
Sven Lukas, Henrik Rother
}

How to cite:

Kurzfassung:

LukAs, S. \& Rother, H. (2016): Moränen versus Till: Empfehlungen für die Beschreibung, Interpretation und Klassifikation glazialer Landformen und Sedimente. - E\&G Quaternary Science Journal, 65 (2): 95-112. DOI: 10.3285/eg.65.2.01

Die glazialgeomorphologische und -sedimentologische Terminologie hat in den vergangenen zwei Jahrzehnten international eine starke Weiterentwicklung erfahren und die Nutzung der Begrifflichkeiten folgt seit geraumer Zeit einheitlichen Richtlinien. Grundsätzlich ist bei der Aufnahme glazialer Ablagerungen auf eine saubere Trennung zwischen Beschreibung und Interpretation zu achten, insbesondere sollten geomorphologische Begrifflichkeiten stets klar von sedimentären Prozessen und den sedimentären Produkten unterschieden werden, um eine terminologisch-interpretative Vermengung von Form (Landform) und Inhalt (Sediment) zu vermeiden. Glaziale Sedimente sollten zunächst ausschließlich aufgrund ihrer lithofaziellen Eigenschaften und unter Nutzung strikt lithologischer Begriffe (wie Diamikton, schräggeschichtete Sande, laminierte Schluffe etc.) beschrieben werden. Erst im nächsten Schritt, und nach eingehender Untersuchung, sollten genetische Begriffe (wie Till, Schmelzwassersande, glaziolimnische ${ }^{1}$ Warvensedimente etc.) zur Interpretation der zuvor beschriebenen Einheiten genutzt werden. Die empfohlene Trennung glazialgeomorphologischer und -sedimentärer Begriffe ist bis heute im deutschsprachigen Raum nicht immer gewährleistet. Dies betrifft v.a. den Begriff der ,Moräné, der einerseits als sedimentäre Sammelbezeichnung für glaziale Ablagerungen verschiedenster Herkunft dient (z.B. ,Moränenmaterial'), anderseits aber auch den durch den Gletscher aktuell transportierten Gesteinsschutt beschreibt (z.B. ,Obermoräne'). Desweiteren wird der Moränenbegriff gleichermaßen sowohl für die Ansprache glazialer Landformen (z.B. Endmoräne) als auch für die Beschreibung von Eigenschaften des Geschiebespektrums genutzt (z.B. Lokalmoräne). Diese Praxis führt nicht nur bei Einsteigern zu Verwirrungen, sondern erschwert auch die Verständigung unter Fachleuten, da diese multifunktionale Nutzung des Moränenbegriffs international seit geraumer Zeit nicht mehr üblich ist. Weitere terminologische Probleme ergeben sich aus den voneinander abweichenden Nomenklaturansätzen, die innerhalb der verschiedenen deutschsprachigen Staaten im Gebrauch sind, sowie der Praxis, dass z.B. in Deutschland quartärgeologische Aufnahmen in den Aufgabenbereich der einzelnen Bundesländer fallen und damit eigene begriffliche Traditionen fortbestehen.

Der vorliegende Artikel hat das Ziel, einen systematischen Überblick über die Genese glazialer Sedimente zu liefern und Empfehlungen für die zukünftige Beschreibung, Benennung und Interpretation solcher Sedimente in der deutschsprachigen Literatur zu liefern, die den internationalen Definitionen entsprechen. Der Begriff, ,Moräne', einschließlich der Variante ,Grundmoräne', sollte fortan lediglich für die Einordnung glazialer Landformen bzw. Landformenvergesellschaftungen verwendet werden, jedoch nicht für die Ansprache glazialer Sedimente. Letztere sollten künftig erst nach genauerer lithologischer Beschreibung und nach den hier definierten diagnostischen Kriterien benannt und geogenetisch interpretiert werden. Die in diesem Artikel präsentierte Zusammenstellung der wichtigsten Kriterien für eine sichere Unterscheidung diverser glazialer Diamikte richtet sich nach dem aktuellen internationalen Forschungsstand. Danach wird ein primärer Till als ein ausschließlich subglaziales und durch direkte Ablagerung vom Eis gebildetes Sediment definiert. Ein solcher in der ,Traktionszone' eines basal gleitenden Gletschers entstandener Till wird als ,subglazialer Traktionstill' (engl. subglacial traction till) bezeichnet. Traktionstills sind von gletscherüberfahrenen prä-existenten Sedimenten (z.B. deformierte Schmelzwassersande) zu unterschieden, welche künftig als ,Glaziotektonit' (engl. glaciotectonite) angesprochen werden sollten und nicht zu den primären Tills sensu stricto gerechnet werden. Eine Weiterverwendung der älteren, stark prozessspezifisch geprägten Till-Begriffe wie Lodgement till und Deformation till ist nach aktuellem Forschungsstand nicht mehr sinnvoll, da zwischen beiden Tilltypen in der Realität ein genetisches Prozesskontinuum besteht und keine gesicherten diagnostischen Kriterien für eine zuverlässige Einzelansprache im Gelände vorliegen.

Andere diamiktische Sedimente, die häufig in glazialen Ablagerungsräumen auftreten und meist supra- bzw. proglazialer Herkunft sind, sollten nicht als Till, sondern nach dem jeweils dominanten Ablagerungsprozess benannt werden (z.B. Schlammstrom(-ablagerung), debris flow (deposit); Abtropfdiamikt, dropstone diamict). Unter Anwendung dieser Kriterien stellen die Autoren fest, dass subglazial gebildeter Till weit weniger verbreitet ist als bislang angenommen. Vor allem glaziale Landformen wie Endmoränen enthalten bei genauer Betrachtung nur selten größere Anteile primärer glaziale Sedimente (Till). Stattdessen setzen sie sich zumeist aus einer Vielzahl verschiedenartiger und deformierter Sedimente zusammen, die im ehemaligen Gletschervorfeld abgelagert und im Zuge eines Vorstoßes zusammengestaucht wurden.

${ }^{1}$ Anmerkung zur Bildung von Wortderivaten des Stammes glazi-
In der deutschen und englischsprachigen Fachliteratur finden sich sowohl glazi- als auch glazio-als Wortderivate von lat. glacies
,Eis' (z.B. glazigen / glaziogen und glacitectonite / glaciotectonite). In vielen Texten werden beide Formen gleichzeitig genutzt
(z.B. Glaziologie, aber glazigen). Funktional dient das -o- in Glaziologie als Verbindungsvokal zwischen dem lateinischen Wort-
stamm glazi- und dem griechischen Suffix -logie. Da glazi- in einem Vokal endet, wäre das verbindende -o- streng genommen
nicht nötig, es wird aber in vielen glazialen Termini genutzt (z.B. in Glaziologie, glaziologisch, glaziofluvial). Andererseits finden
sich viele Textbeispiele von Wortzusammensetzungen bei denen die Autoren auf das -o- verzichten (z.B. glazigen, glazilacustrin,
glazifluvial). Die verschiedenen Handhabungen in der Wortbildung sind verwirrend und sollten im Interesse einer einheitlichen 
Regelung (generell mit oder ohne Verbindungsvokal -o-) überdacht werden. Da das verbindende -o- in der Bezeichnung des Faches Glaziologie allgemein genutzt wird (die Alternative wäre ansonsten Glazilogie), empfehlen wir die Einfügung des Verbindungsvokals zukünftig auf alle mit glazi-zusammengesetzten Worte zu übertragen (d.h. glaziofluvial, glaziogen, Glaziotektonik etc.).

Moraines versus till: Recommendations for the description, interpretation and classification of glacial landforms and sediments

Abstract:

Internationally, the description and interpretation of glacial sediments and landforms largely follow a set of uniform guidelines and terminology. Sediments are normally described according to their lithofacies characteristics (e.g. diamicton, sorted sands), and only after closer inspection and investigation are genetic terms applied (e.g. till, glaciofluvial outwash). Mixing of sedimentological and geomorphological terminology does not occur. In German-speaking countries, however, the term moraine is used for glaciogenic sediments and landforms such as end moraines and also adopted for till plains ("ground moraine landscapes", "old/young morainic landscapes" etc.). Similar traditions of the latter kind are sometimes found as a relict in Scandinavian texts, and an equally profound mixing of terms is found in much of the French literature. The authors argue here that this mixture not only leads to unnecessary confusion for students but also makes international communication more difficult, especially when the terminological inconsistencies are as extensive and non-systematic as in the German community at present. The present paper presents a systematic overview of the state-of-the-art of till terminology, thereby providing the necessary background information for a useful description and interpretation of field evidence for communicating results in German and hopefully aiding more efficient communication of German Quaternary geologists and geomorphologists internationally. The aim is to provide a sedimentological terminology that is in agreement with international standards and can readily be distinguished from geomorphological vocabulary. The authors recommend that usage of the term ground moraine, for example, is restricted to certain landform associations encountered in NW Central Europe, and excluded from use when discussing sediments. For primary glaciogenic sediments sensu stricto the term till should be used only where appropriate sedimentological evidence exists. A process-based subdivision of till types (e.g. deformation, lodgement till) is not useful in most cases, because as a community we do not have the tools to reliably distinguish such processes at a macro-scale. This recognition has led to the creation of the highly useful umbrella term subglacial traction till and its distinction from overridden primary sediments that are termed glaciotectonite. The present contribution translates the older terminology to the internationally-accepted terminology that follows the scientifically-robust approach of splitting descriptive terminology - based on a lithofacies approach (e.g. diamicton) - from the latter interpretative stage (e.g. subglacial traction till, debris flow deposit). The authors present translations of the different till schemes that have existed through time and link these to the current state of the art, citing several examples and clear diagnostic criteria to distinguish various types of diamictic sediments. This contribution stresses that the majority of diamictons encountered in glacial environments, especially in ice-marginal settings, are not usually and certainly not automatically subglacial traction tills.

Keywords:

Till, subglazialer Traktionstill, glaziale Sedimente, Sedimentologie, Quartärgeologie, Diamikton, Lithofaziesaufnahme, Moräne, Grundmoräne, Terminologie, subglacial traction till, glacial sediments, sedimentology, Quaternary Geology, diamicton, debris flow, lithofacies approach, moraines

Addresses of authors: Sven Lukas*, School of Geography, Queen Mary University of London, Mile End Road, London E1 4NS, UK. E-Mail: S.Lukas@ qmul.ac.uk, Tel. +44 207882 8417; Henrik Rother, Institut für Geographie und Geologie, Ernst-Moritz-Arndt-Universität Greifswald, Friedrich-Ludwig-Jahnstr. 17a, 17489 Greifswald, Germany. * corresponding author

\section{Einleitung}

Primäre glaziale Sedimente, im Englischen als ,Till', im Deutschen bislang oft als ,Moräne bezeichnet, umfassen eine Reihe zumeist schlecht sortierter Diamikte mit Anteilen aus fast allen Korngrößenfraktionen. Diese höchst variable Zusammensetzung - keine zwei Tills sind identisch - erschwert die klare Definition dieser Sedimentgruppe und bedingt die z.T. sehr unterschiedlichen hydrogeologischen und baugrundgeologischen Eigenschaften dieser Ablagerungen (PiOTROwski 1992, BELl 2002, EvANS et al. 2006, Griffiths \& MARTIN 2016). Nichtsdestotrotz ist das Verständnis dieser Gruppe von Sedimenten von herausragender Bedeutung, nicht zuletzt weil glaziale Ablagerungen weltweit über $30 \%$ der Kontinente bedecken (GoldTHWAIT 1971, Benn \& Evans 1998, 2010).

Aus Sicht der internationalen Quartärgemeinschaft ist die korrekte Ansprache und Interpretation sedimentärer Merkmale sowie der Genese glazialer Sedimente von großer Wichtigkeit für die Rekonstruktion glazialer Prozesse, wobei eine einheitliche und klar definierte sedimentologische Terminologie unabdingbar ist (Dreimanis 1988, PioTROWSKI 1992, VAN DER MEER et al. 2003, EvANS et al. 2006, Benn \& Evans 2010, Griffiths \& Martin 2016). Die Not- wendigkeit der Entwicklung einer modernen Nomenklatur auf Basis des aktuellen glazialgeologischen Kenntnisstandes besteht auch für den deutschen Sprachraum. Ziel dieser Bestrebung ist es, Beiträge der deutschsprachigen Forschung zu internationalen quartärwissenschaftlichen Projekten zu erleichtern, und durch die Schaffung einheitlicher Kriterien für die regionale Quartärkartierung zu einer effektiveren Verständigung zwischen z.B. geologischen Landesbehörden und universitären Einrichtungen beizutragen.

Die Untersuchung glazialer Sedimente hat eine fast zweihundertjährige Tradition in den Geowissenschaften, wobei sich über diesen langen Zeitraum ganz unterschiedliche Ansätze in den verschiedenen geologischen Teildisziplinen und Nationalstaaten entwickelt haben. Im Hinblick auf die Beschreibung und Interpretation glazialer Ablagerungen orientiert sich die Quartärgeologie im deutschsprachigen Raum zwar durchaus an glazialsedimentologischen Ansätzen, jedoch folgt die Terminologie in vielen Fällen nicht den international üblichen Standards. Gleichzeitig findet in der Physischen Geographie häufig eine aus der deutschen Bodenkunde entliehene Methodik und Terminologie zur Beschreibung und Interpretation Anwendung, was u.a. zur Folge hat, dass die von der deutschsprachigen Quartärgemeinschaft derzeit genutzte sedimentologische 
Nomenklatur nach wie vor mit stark geomorphologischen Konnotationen behaftet ist (Tabelle 1). Anhand dieser Beispiele wird deutlich, dass der Begriff ,Moräne‘, bzw. verschiedene davon geformte Derivate, bis heute sowohl für glaziale Landformen als auch für primär an der Gletschersohle gebildete Sedimente genutzt wird, obwohl die klare begriffliche Trennung seit nunmehr über zwei Jahrzehnten empfohlen wird (z.B. Piotrowski 1992, Lukas 2004, WinkLER 2009, EHLERS 2011). Die daraus resultierende begriffliche Dichotomie führt nicht nur zu Schwierigkeiten in der fachlichen Kommunikation zwischen benachbarten Disziplinen, sondern auch dazu, dass sich deutsche und internationale Fachleute terminologisch voneinander entfernt haben und im Gelände, z.B. vor glazialen Aufschlüssen, nur unter Schwierigkeiten präzise miteinander kommunizieren können.

Mit dem vorliegenden Beitrag möchten die Verfasser auf eine Reihe von Problemen hinweisen, die sich aus der in Deutschland noch üblichen Vermischung geomorphologischer und sedimentologischer Termini ergibt, und gleichzeitig einen konstruktiven Vorschlag für eine zukünftige Nomenklatur unterbreiten. Um damit sowohl fortgeschrittene Wissenschaftler als auch praktisch arbeitende Geologen und Studenten der Quartärgeologie und Quartärwissenschaften allgemein zu erreichen, beginnen wir mit einer kurzen Darstellung der historischen Entwicklung der Tillforschung. Im folgenden Hauptteil des Beitrages fassen wir dann, veranschaulicht anhand von Beispielen, den aktuellen Wissensstand zur Genese und Ansprache glazialer Sedimente zusammen. Dabei werden verschiedene historische Konzepte und die daraus resultierenden Termini in ihrem ehemaligen Zusammenhang erläutert, sodass auch heute obsolete Termini der älteren Fachliteratur erklärt und verständlich werden; ein entsprechender Übersetzungsschlüssel wird in diesem Rahmen mitgeliefert.

\section{Historische Entwicklung der Moränen- und Till- Nomenklatur}

Die nach der heutigen Nutzung ambivalente Bedeutung des geomorphologischen und sedimentologischen Moränenbegriffs hat seinen Ursprung in der Verzahnung des französischen und deutschen Sprachgebrauchs im 18. und 19. Jahrhundert (von BöHMERsheim 1901). So war der Begriff ,Moräne zur Zeit der ersten wissenschaftlichen Beschreibungen ein alltagssprachlicher Terminus der deutschsprachigen Alpenbewohner, der ausschließlich zur Beschreibung wallartiger Sedimentakkumulationen am Eisrand von Talgletschern benutzt wurde, und auch im französischen alpinen Sprachraum wurde der Begriff , moraine in diesem Sinne im 18. Jahrhundert verwendet (DE SAUSSERE 1779).

Die Einführung des Moränenbegriffs in die deutsche Fachliteratur geht vermutlich auf AgAssiz (1838) zurück, der diesen jedoch auch auf den im supraglazialen Transport befindlichen Schutt aktiver Gletscher anwendete (dieser war bis dahin mit dem in der Gegend um Grindelwald gebräuchlichen Begriff ,Guffer bezeichnet worden: siehe KuHn 1786, in von Böhmersheim 1901). Damit kam es bereits in Frühphase der Glazialforschung zur mißverständlichen Vermischung des ursprünglichen Landformenbegriffes mit dem supraglazialen Schuttkörper (vgl. LukAs et al. 2007). Die darüber hinausgehende, und bis heute im

Tab. 1: Beispielhafte Zusammenstellung in der Literatur gebräuchlicher, unterschiedlicher und oft synonym nebeneinander verwendeter Bezeichnungen für primäres glaziogenes Sediment.

Tab. 1: Examples of the different terms emplyoed to refer to primary glaciogenic sediments. Note that these are often used alongside each other and partly synonymously, even in the same contribution.

\begin{tabular}{|c|c|c|}
\hline Terminologie & Bemerkung / Erklärung ${ }^{1}$ & Quelle[n]/Autor[en] \\
\hline Moräne & $\begin{array}{l}\text { Bezeichnung sowohl für Sediment als } \\
\text { auch für Landform }\end{array}$ & $\begin{array}{l}\text { EHLERS 1990, 1994, 2011, HeRget 1997, KüHn et al. } \\
\text { 2002, Kamp \& HaSeroth 2002, Zepp 2002, HaNTKE } \\
\text { \& WaGner } 2004\end{array}$ \\
\hline $\begin{array}{l}\text { Grundmoräne / Grundmoränen- } \\
\text { landschaft }\end{array}$ & $\begin{array}{l}\text { Bezeichnung für verschiedene unter dem } \\
\text { Gletscher abgelagerte Sedimente und } \\
\text { die daraus aufgebauten Landformen, die } \\
\text { eine ehemalige Eisbedeckung anzeigen }\end{array}$ & $\begin{array}{l}\text { GlatthaAr \& LiedtKe 1981, LiedtKe 1990, Dahm et } \\
\text { al. 1985, Braun 1987, Driesen 1990, Ehlers 1990, } \\
\text { Grube 1990, van Husen 1990, Herget 1997, 1998, } \\
\text { Karte 1998, KüHn et al. 2002, Kuhle 2002, Zepp } \\
\text { 2002, Winkler 2009 }\end{array}$ \\
\hline Geschiebelehm / -mergel & $\begin{array}{l}\text { Lithofazielle Beschreibung diamiktischer } \\
\text { glazialer Sedimente unterschiedlichen } \\
\text { Karbonatgehaltes [entkalkt / kalkhaltig] }\end{array}$ & $\begin{array}{l}\text { Braun 1987, Herget 1997, Kalser et al. 2002, Zepp } \\
\text { २००2 }\end{array}$ \\
\hline $\begin{array}{l}\text { Moränenmaterial / -sedimente / } \\
\text {-ablagerungen /-schutt }\end{array}$ & s. Moräne \& Grundmoräne & $\begin{array}{l}\text { Ehlers 1990, 1994, 2011, Grube 1990, van Husen } \\
\text { 1990, GlatthaAr 1998, WinkLer \& Hagedorn } 1999\end{array}$ \\
\hline Lokalmoräne & $\begin{array}{l}\text { Moränensedimente, deren Zusammen- } \\
\text { setzung [Geschiebespektrum, Korngrös- } \\
\text { senfraktionen etc.] durch lokal anste-- } \\
\text { hende Gesteine dominiert wird }\end{array}$ & Glatthaar \& Liedtke 1981, Braun 1987, Ehlers 2011 \\
\hline Jung- bzw. Altmoränenlandschaft & $\begin{array}{l}\text { Während der letzten bzw. vorletzten oder } \\
\text { älteren Vereisung vergletschertes Gebiet }\end{array}$ & $\begin{array}{l}\text { LiedtKe 1981, 1990, GruBe 1990, KüHn et al. 2002, } \\
\text { Zepp } 2002\end{array}$ \\
\hline
\end{tabular}

${ }^{1}$ Bedeutung aus dem Kontext der betreffenden Quellen hergeleitet, sofern nicht explizit definiert. 
Deutschen gebräuchliche, Anwendung des Wortes ,Moräne' für bereits abgelagerte subglaziale Sedimente geht auf MARTINS (1842, in VON BöHMERSHEIM 1901) zurück, der als erster auch Sedimente in subglazialer Position mit, moraine profonde bezeichnete (von BöHmersheim 1901). Diese Begriffsnutzung ist im historischen Kontext durchaus nachvollziehbar, denn die Sedimentologie war als eigenständige Disziplin zu dieser Zeit kaum entwickelt (OKADA \& KenYoN-SMith 2009) und es bestand verständlicherweise noch kein Bewußtsein für die Problematik, die sich aus der doppeldeutigen Begriffsanwendung ergeben sollte. Im Gegensatz zum deutschen Sprachgebrauch bestand im angloamerikanischen Raum von Beginn an - bis auf wenige Ausnahmen - eine klare Trennung der Termini für glaziale Sedimente einerseits und der für glaziale Landformen andererseits (vgl. Dreimanis 1988, Benn \& Evans 1998, 2010, Evans \& Benn 2004a, Bennett \& Glasser 2009). Die Erforschung vor allem derzeit vergletscherter Regionen hat in den letzten Jahrzehnten zu einem wesentlich besseren Verständnis glazialer Prozesse geführt und die Entwicklung eines einheitlichen Ansatzes sowie einer Terminologie nach sich gezogen, die unten detailliert erläutert werden.

\section{3 Überblick zur aktuellen glazialsedimentologischen Terminologie 3.1 Diamikton}

Wie bei allen sedimentologischen Aufnahmen ist auch bei der Beschreibung glazialer Sedimente auf ein systematisches Vorgehen zu achten, wobei jede genetische Interpretation auf möglichst viele Daten zu stützen ist (BENN \& Evans 2004). Für unsortierte und häufig matrixdominierte Sedimente, die in glazialen Milieus oft zur Ablagerung kommen, ist der lithologische Begriff ,Diamikton' (engl. diamicton) zu verwenden. Damit werden auf neutrale Weise solche Sedimente beschrieben, die aus einer weitgehenden Vermischung von zwei oder mehreren granulometrischen Hauptbestandteilen hervorgegangen sind, und die in vielen Fällen bimodale Häufigkeitsmaxima (meist in der Schluff- und Sandfraktion) aufweisen, daneben jedoch auch Komponenten aller anderen Korngrößenklassen enthalten können (Frakes 1978, Eyles et al. 1983, Evans \& BenN 2004b, Benn \& Evans 2010). Diamikte werden als korngestützt (clast-supported) oder matrixgestützt (matrixsupported) klassifiziert und sind meist ungeschichtet (massive diamicton), können jedoch auch grobe bzw. schwache Schichtungsmerkmale aufweisen (stratified diamicton). Aussagen zum Grad der Verfestigung wird häufig eine hohe Bedeutung zugemessen, da subglazial gebildete Diamikte aufgrund der Eisauflast meist überkonsolidiert (overconsolidated) sind, was von einigen Autoren auch als ,Vorbelastung' bezeichnet wird (z.B. ScHLÜCHTER 1999). Angaben zur Konsistenz dieser Materialien können daher relevante Informationen zum Ablagerungsmilieu liefern (z.B. subglazial, subaerisch, subaquatisch), und daher schlagen z.B. KRÜGER und KJÆR (1999) folgende vierstufige Einteilung vor: (1) nicht kompaktiert (loose, non-compacted), (2) bröselig, leicht zu graben (friable, easy to excavate), (3) fest, schwer zu graben (firm, difficult to excavate) und (4) extrem fest (extremely firm).
Ein häufig auftretender Fehler, nicht nur bei Studierenden, ist der quasi-automatische Sprung vom lithologischdeskriptiven Term ,Diamikton“ zum genetischen Begriff ,Till'. Eine solche vorschnelle genetische Schlussfolgerung ist jedoch in jedem Falle zu vermeiden, da Diamikte auch in glazialen und nicht-glazialen Ablagerungsräumen durch ganz verschiedene sedimentäre Prozesse entstehen können (siehe OWEn 1994, READING 1996, LukAs 2004). So werden zahlreiche Diamikte als subaerische oder subaquatische Schlammströme gebildet oder entstehen durch periglaziale Verwitterungsprozesse. Selbst im Kontext glazialer Sedimentabfolgen ist die häufig anzutreffende automatische Parallelisierung der Begriffe ,Diamikton' und ,Till' ohne ausreichende Befundlage spekulativ, und entspräche der vorschnellen Interpretation aller massigen Siltablagerungen in Periglazialgebieten als Löss, ohne andere genetische Möglichkeiten in Betracht zu ziehen.

In diesem Zusammenhang sei auch darauf hingewiesen, dass die deutschen Begriffe ,Geschiebemergel' und ,-lehm' nicht für eine genetisch neutrale Sedimentbeschreibung geeignet sind und damit auch nicht als Ersatz für den deskriptiven Begriff ,Diamikton“ dienen können. Das terminologische Problem besteht darin, dass die Ansprache von Klasten als ,Geschiebe' im quartärgeologischen Sprachgebrauch bereits ein Sediment glazialen Ursprungs festlegt. Hinzu kommt, dass die Zusätze -mergel und -lehm die stoffliche Zusammensetzung spezifizieren (Vorhandensein von Karbonat bzw. Fehlen durch postsedimentäre Entkarbonatisierung) und damit nicht für eine Bestimmung der faziellen Genese geeignet sind (vgl. Piotrowski 1992, LuKAS 2004). Aus diesen Erwägungen ergibt sich, dass der Begriff ,Diamikton' für eine explizit deskriptive Ansprache von schlecht sortierten Korngemischen vorzuziehen ist, da es sich hierbei um einen lithologisch neutralen, nicht-genetischen Begriff handelt. Im Weiteren folgt ein zusammenfassender Überblick der verschiedenen Till-Typen sowie der wichtigsten Charakteristika, die zu ihrer Unterscheidung im Gelände und Labor herangezogen werden können.

\subsection{Tills}

Das Wort ,Till' hat seinen etymologischen Ursprung in der Alltagssprache Schottlands, wo er lange vor der Akzeptanz der Glazialtheorie benutzt wurde, um einen Boden „aus steinigem Ton“ zu beschreiben (Flint 1971: 148). Aufgrund der herausragenden Bedeutung Schottlands für die Entwicklung der frühen Glazialgeologie (z.B. GEIKIE 1863) fand der Begriff frühzeitig Einzug in die angloamerikanische Glazialterminologie. In der Literatur werden unterschiedliche Arten von Till aufgeführt. Im Folgenden werden die gängigsten Arten beschrieben, diskutiert und sinnvolle Übersetzungen in die deutsche Terminologie vorgeschlagen. Dabei werden auch solche Till-Typen angeführt, die heute größtenteils nicht mehr als eigenständige Tills anerkannt werden. Für die INQUA Kommission zur ,Genese und Lithologie quartärer Ablagerungen“ definierte Dreimanis (1988: 41) einen Till sensu lato als ein primäres glaziales Sediment, welches „durch das unmittelbare Herauslösen von Schutt aus dem Gletscher durch basales Absetzen, Ausschmelzen oder Sublimation entsteht“. Es ist anzumerken, dass die ursprüngliche Till-Definition damit in 
gewissem Umfang Resedimentations- und Aufarbeitungsprozesse mit einbezog, auch wenn diese bei der Tillgenese keine dominante Rolle einnehmen sollten (DrEIMANIS 1988: 41f.). Dagegen schließen die neueren Definitionen von Till explizit die von Dreimanis (1988) noch einbezogene Resedimentation und Aufarbeitung bei der Tillgenese aus (LAwson 1988, Hicock 1990, BenN \& Evans 1996, 1998, 2010, Evans \& BENN 2004b, Evans et al. 2006, LukAs et al. 2012). Subaquatische Tills, ursprünglich als Ablagerung in wassergefüllten subglazialen Becken oder unter Eisschelfen definiert (Dreimanis 1988, PiOTROWSKi 1992), werden aufgrund der heute enger gefassten Definition, die das Sedimentieren durch eine Wassersäule ausschließt, nicht mehr zu den Tills gerechnet. Sofern möglich, sollten diese Sedimente nach den jeweiligen Geneseprozessen weitergehend klassifiziert werden, wobei Diamikte in diesem Ablagerungsraum meist als subaquatische Schlammstromablagerungen (engl. subaqueous debris flow) oder durch das Ausregnen von Material aus der aufschwimmenden Gletscherbasis oder aus Eisbergen entstehen (Abtropfdiamikton, engl. undermelt diamicton, dropstone diamicton).

Innerhalb des oben beschriebenen Definitionsrahmens für Till, der die Resedimentation sowie die Aufarbeitung durch fließendes Wasser kategorisch ausschließt, wurden die folgenden drei primären Tilltypen unterschieden:

(a) Absetz- oder Setztill (engl: Lodgement till) entsteht durch das Herauslösen von Material aus dem basalen Gletschereis, wobei Partikel, Klasten oder Sedimentschollen durch ,Aufschmieren' am Gletscherbett zur Akkumulation teils mächtiger Sedimentpakete führen kann (Piotrowski 1992, BENn \& Evans 1998). Physikalisch erfolgt das Absetzen von Material aus der Gletschersohle entweder, wenn die auf die Klasten wirkenden retardierenden Reibungskräfte am Gletscherbett die Adhäsionskräfte des Eises übersteigen oder wenn Material durch basales Druckschmelzen freigesetzt wird. Aufgrund seiner Entstehung an der Basis gleitender Gletscher ist ein Setztill in vielen Fällen sehr stark verfestigt (engl. overconsolidated) und von massiger Erscheinung. Verbreitet finden sich makro- und mikroskopische, subhorizontal orientierte Scherklüfte, die glaziotektonisch angelegt sind und im Zuge des Austrocknens bzw. der Verwitterung als Kontraktions- oder Entlastungsklüfte sichtbar werden (Abb. 1a-c). Geschiebe im Lodgement Till sind zumeist gut kantengerundet, zeigen oft geschrammte bzw. polierte Oberflächen und sind durch Detersion, Klast-Rotation und Mikro-Detraktion geometrisch oft in ,Projektilform' entwickelt (engl. bullet-shaped clast; Abb. 1d). Weitere ehemals gängige Kriterien für die Identifikation von Setztills finden sich in den Zusammenstellungen von Piotrowski (1992), Krüger (1994) und BenN \& Evans (1998). Allerdings ist es nach heutigem Kenntnisstand und auf Grundlage moderner mikromorphologischer Untersuchungen zweifelhaft, ob reine Setztills in der Realität überhaupt auftreten (EvANs et al. 2006; siehe Diskussion unten).

(b) Basaler Ausschmelztill (engl. meltout till) entsteht subglazial durch das Ausschmelzen schuttreichen basalen Eises von stagnierenden oder nur langsam fließenden Gletschern (BENN \& Evans 1998). Häufige Strukturen umfassen grobe Schichtungsmerkmale häufig mit einer Wechsellagerung von diamiktischen und geringmächtigen sortierten Lagen sowie der symmetrischen Drapierung eingeschlos- sener Geschieben durch o.g. Lagen (PAul \& Eyles 1990, PIOTROwski 1992). Manche Autoren zählen auch Sedimente, die durch das supraglaziale Ausschmelzen von Schutt auf stagnierenden Eiskörpern entstehen, zu den Ausschmelztills (supraglacial meltout till; z.B. Boulton 1970, DreimANIS 1988) obwohl gravitative Materialverlagerungen häufig maßgeblich an der Genese dieser Ablagerungen beteiligt sind. Generell ist anzumerken, dass die im Austauprozess entstehenden Schmelzwässer mit hoher Wahrscheinlichkeit zu einer sekundären Aufarbeitung der ausschmelzenden Materialien führen, wodurch sich ein Widerspruch zur INQUA Definition von primärem Till ergibt (,... abgelagert vom Gletschereis ohne maßgebliche Aufarbeitung durch fließendes Wasser", Dreimanis 1988). Aufgrund des anzunehmenden Einflusses sekundärer Aufarbeitungsprozesse ist insgesamt von einem nur geringen Erhaltungspotential der primären Ablagerungsstrukturen (wie z.B. Stratifizierung) in subglazialen und supraglazialen Ausschmelztills auszugehen (Paul \& Eyles 1990, Lukas et al. 2005, 2007, Evans et al. 2006, LuKas 2007, 2011, Evans 2009).

Der Sublimationstill (engl. sublimation till) galt als eine besondere Variante des Ausschmelztills, da hierbei das im Gletschereis transportierte Material durch Sublimation frei wird, d.h. durch den direkten Phasenübergang von Eis zu Wasserdampf, ohne eine zwischengeschaltete Verflüssigung. Direkte Beobachtungen haben gezeigt, dass sich Ablagerungsprozesse durch Submilation sehr langsam vollziehen und nur zu geringmächtigen Tilllagen (dm-Bereich) führen; zudem ist dieser Tilltyp auf Gletscher extrem kalter und arider Klimazonen (z.B. rezent in der Antarktis) beschränkt (LundQvist 1989, BENN \& EvANS 1998). Aus diesen Gründen ist es unwahrscheinlich, dass Sublimationstills in größerer Verbreitung und Mächtigkeit in den während des Pleistozäns vereisten Gebieten der mittleren Breiten auftreten.

(c) Deformationstill (engl. deformation till): Definiert als Sediment, „das durch Scherung in einem subglazialen Deformationshorizont weitestgehend disaggregiert und zu großen Teilen homogenisiert wurde“ (BENN \& Evans 1998: 390). Der subglaziale Deformationshorizont (engl. deforming bed) beschreibt dabei eine max. $2 \mathrm{~m}$ mächtige wassergesättigte Lockersedimentzone aus Till oder eisüberfahrenen Sedimenten, welche an der Basis vieler temperierter Gletscher zu finden ist. Die in dieser Zone auftretenden hohen Porenwasserdrücke reduzieren die Kohäsion innerhalb der subglazialen Sedimente und führen, in Kombination mit den Reibungseffekten der basalen Eisbewegung, zu einer durchgreifenden mechanischen Homogenisierung der subglazialen Sedimente (= ,deforming layer $\left.{ }^{6}\right)$. Prinzipiell lässt sich der Prozess mit dem Um- bzw. Unterrühren verschiedener Komponenten zu einer homogenen ,Paste“ vergleichen; mögliche präexistente sedimentäre Strukturen werden dabei weitestgehend zerstört. Neuere Prozessstudien an rezenten Gletschersohlen belegen, dass einzelne vom Eis in das Sediment hineinragende Unregelmäßigkeiten (z.B. angefrorene Klasten) durch ihre ,pflügende“ Bewegung eine wesentliche Rolle bei der physischen Homogenisierung subglazialer Lockersedimente spielen (z.B. TulACZYK et al. 2000, 2001, EvAns et al. 2006).

In der älteren Literatur wird der Deformationsstill zumeist als massig-schichtungsloser Diamikt beschrieben, 

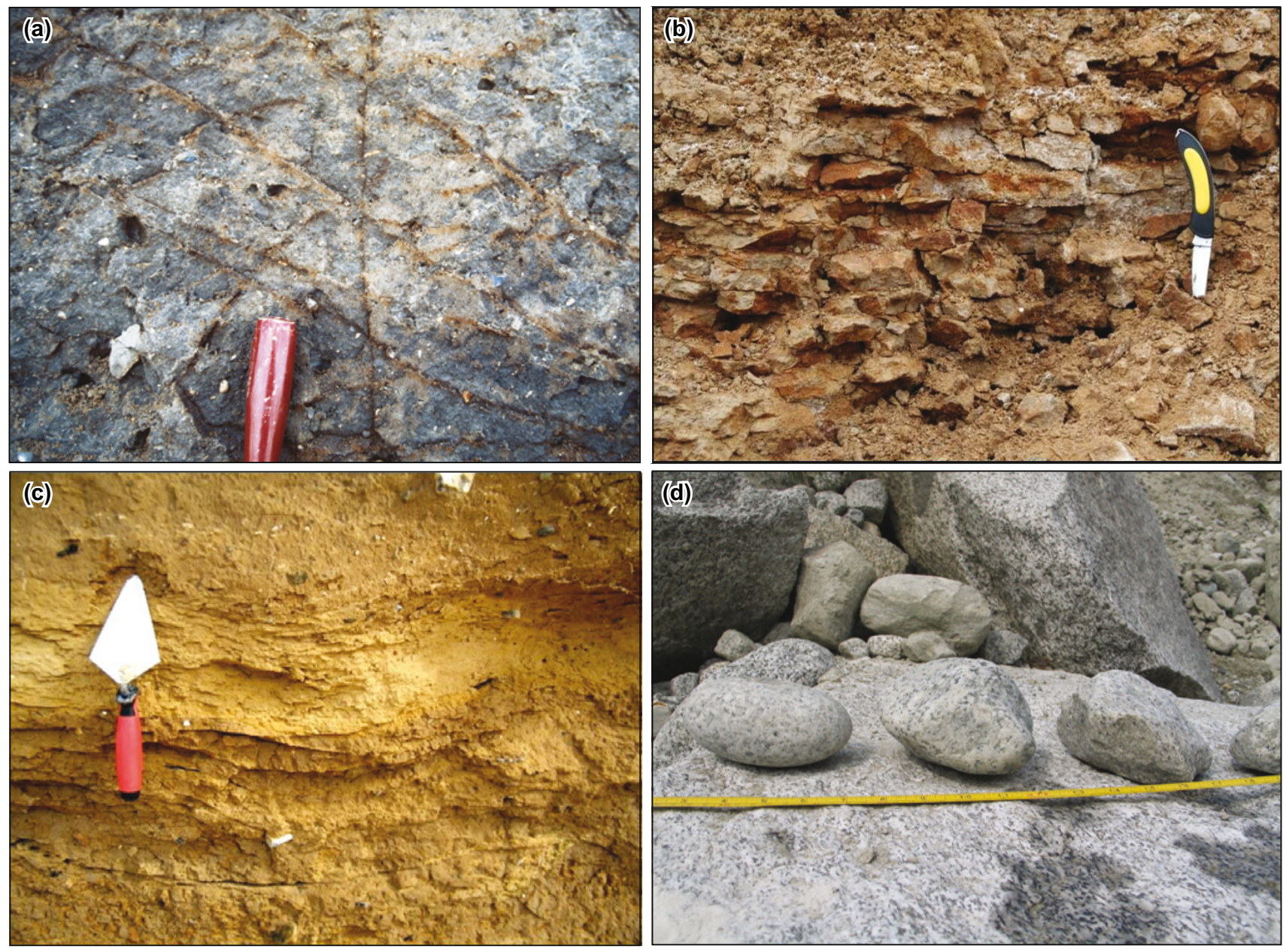

Abb. 1: (a)-(c): Verschiedene Ausprägungen von Klüftung in Diamikten, in diesem Fall sämtlich subglaziale Traktionstills. (a): Liefdefjord, Dänemark; (b)-(c): Insel Poel, Deutschland; (d): Verschiedene Rundungsgrade geschoßartiger Geschiebe, die aus einem subglazialen Traktionstill isoliert wurden. Man erkennt, von rechts nach links, die progressive Rundung durch fluviale Prozesse vor der Wiederaufnahme in die basalen Eislagen und damit auch die Mischung von Material unterschiedlicher Transportstadien (,Reife '). Hornkees, Österreich.

Fig. 1: $(a-c)$ : Examples of the different character of fissility in diamicts, in this case subglacial traction tills. (a) Liefdefjord, Denmark; (b-c): Isle of Poel, Germany; (d): different degress of rounding in bullet-shaped clasts extracted from subglacial traction till. Progressively more intense fluvial reworking prior to re-incorporation of these clasts into the basal sediment layer is evident from right to left, indicating the mixing of material with very different transport histories ('maturity'). Hornkees, Austria.

wobei zum Teil deutliche Anzeichen für Verfaltung und Scherung, die vor allem an verformten intra-till Sedimentlinsen (streaked-out sediment lenses) sichtbar werden, auftreten können. Ausführliche Darstellungen der für den Deformationstill ehemals verwendeten diagnostischen Merkmale finden sich u.a. bei BENN \& Evans (1996, 1998), vAN Der Wateren (1995), van der WATERen et al. (2000) und Piotrowski et al. (2001). Neuere konzeptionelle Arbeiten zur Klassifikation von Till weichen insofern von früheren Vorstellungen zum Deformationstill ab, als dass für durch aktive Eisbewegung deformierte Lockergesteine (seltener auch Festgesteine), die selbst nicht glaziogenen ${ }^{1}$ Ursprungs sind, der Begriff ,Glaziotektonit' (engl. glaciotectonite) eingeführt wurde (BANHAM 1977, BENN \& Evans 1996). Inbegriffen sind Locker- und Festgesteine, die durch „subglaziale Scherung deformiert wurden, jedoch einige Charakteristika des Ausgangsmaterials beibehalten "(BENN \& Evans 1998: 387). Nach dieser Einteilung werden vom Ursprung her nicht-glaziogene Sedimente, die während eines Eisvorstoßes überfahren und tektonisch beansprucht wurden, von "echtem“ Deformationstill unterschieden (BenN \& Evans 1996, LukAs 2005, 2007, 2012). Entscheidendes Merkmal der
Glaziotektonite ist ein im Vergleich zum Deformationstill geringerer Grad an tektonisch bedingter Homogenisierung, weshalb bei Glaziotektoniten typischerweise Reste ursprünglicher Sedimentstrukturen erhalten sind. Glaziotektonisierter Fels, der im Kontakt mit der Gletscherbasis stand, enthält typischerweise schluffige Einschaltungen und kleinere Felsfragmente in Klüften (Evans et al. 1998, Phillips et al. 2013).

An vielen glazialen Aufschlüssen ist ein Ineinandergreifen verschiedener Tilltypen zu beobachten, wobei es, auch auf kurzer Distanz, zu nahezu stufenlosen Übergängen zwischen zwei Tilltypen kommen kann. Um dies zu erklären, wurde von verschiedenen Bearbeitern (u.a. Нicock 1990, BENN \& Evans 1996) angeführt, dass die grundsätzlichen tillgenetischen Prozesse des ,Ausschmelzens', der ,Setzung und der ,Deformation' nicht strikt voneinander trennbar sind, sondern dass diese vielmehr die konzeptionellen Endpunkte eines Till-Kontinuums bilden (Abb. 2). Damit wird die z.T. erhebliche natürliche Variabilität innerhalb einzelner Tills erklärbar. Durch die Verlängerung des „primären Till-Dreiecks" kann zudem der Einfluss gravitativer Umlagerungsprozesse erfasst werden, die viele primäre Tills 


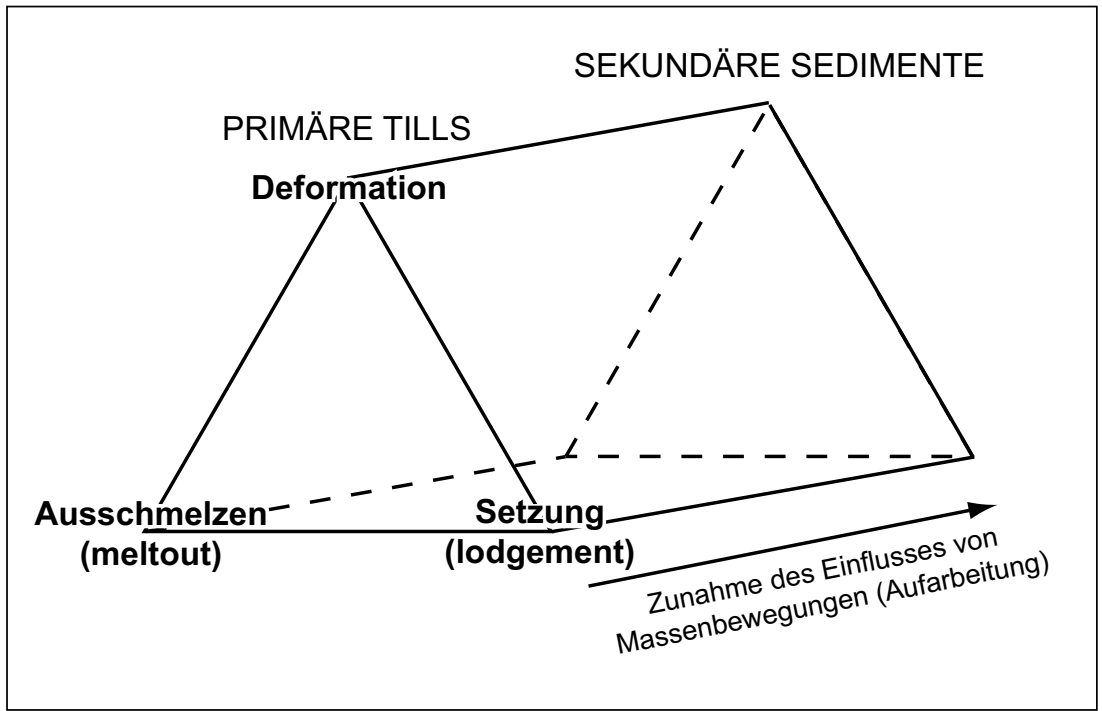

Abb. 2: Das ursprüngliche Till-Prisma nach HICOCK (1990), abgeändert und weiterentwickelt von BENN \& EVANS (1996, 1998). Das Diagramm wurde als konzeptionelle Visualisierungshilfe entwickelt, um die Verknüpfung verschiedener Prozesse bei der Tillgenese darzustellen (,process-product continuum'). Fig. 2: The original till-prism (after HICOCK 1990, modified by BENN \& EVANS 1996, 1998). This diagram was originally developed to visualise the concept of linkages between the different processes that play a role in till genesis ('process-product continuum'). im Zuge des Eisrückzuges und -zerfalls erfahren. Sowohl gravitative Umlagerung als auch Aufarbeitung durch fließendes Wasser führen jedoch zur Transformation in eine durch sekundäre Merkmale dominierte Ablagerung, die dann nicht mehr als Till bezeichnet werden sollte (Нicocк 1990, BENN \& Evans 1996). Aus diesem Grund sind solche glazialen Diamikte, die nach DrEIMANIs (1988) noch zu den Tills gerechnet wurden, in den nachfolgenden Klassifikationen nicht mehr enthalten. Dies betrifft z.B. Ablagerungen, die früher als ,Fließmoräne (flow till) angesprochen wurden, die jedoch heute, je nach Milieu und spezifischem Sedimentationsprozess, entweder den subaerischen, supraglazialen oder subaquatischen Schlammstromablagerungen (engl. subaerial, supraglacial, subaqueous debris flow) zugeordnet werden (LAwson 1988, BENN 1992, KRIENKE 2003, Evans \& Benn 2004a, Lukas 2005, 2007, 2012, PhilLIPS 2006, REINARdy \& LUKAS 2009, Evans \& HARRISON 2014). Gleichsam ist der Glaziotektonit, aufgrund der zumeist erhaltenen Merkmale der Ausgangsfazies, als Zwischenstufe zum vollendeten Deformationstill aufzufassen, und wird demnach nicht zu den Tills sensu stricto gerechnet (BEnN \& Evans 1996, 1998).

In einem der wohl provokativsten Beiträge zur Tillforschung der letzten Jahre argumentieren VAN DER MEER et al. (2003), dass mikromorphologische Untersuchungen eine klare Dominanz deformativer Elemente in allen Tills belegen, und dass mithin auf dieser Maßstabsebene kaum diagnostische Merkmale für einen differenzierbaren sedimentären Ursprung vorliegen. Nach VAN DER MEER et. al. (2003) ergibt sich daraus, dass die bis dahin definierten und oben genannten tillgenetischen Prozesse in ihrer Reinform vermutlich nicht existieren und damit auch nicht Grundlage für eine Differenzierung verschiedener Tilltypen sein kann. Auf Grundlage der mikromorphologischen Hinweise schlussfolgern VAN DER MEER et al. (2003), dass alle Tills in einem subglazialen Deformationshorizont (deforming bed) entstehen. Akzeptiert man diese Neudefinition, so entfallen alle o.g. sedimentären Till-Typen zugunsten eines einzigen strukturell geprägten Tilltyps, dem sogenannten ,Tektomikt'. Aus Sicht vieler anderer Bearbeiter ist jedoch die alleinige Anwendung mikromorphologischer Kriterien bei der Tillansprache, d.h. ohne Hinzuziehung makroskopi- scher Befunde, weder konzeptionell zufriedenstellend noch im Gelände praktikabel. Vielmehr belegen zahlreiche neuere Studien, dass mikro- und makroskopische Ansätze im Verbund genutzt die beste Grundlage für eine präzise genetische Till-Ansprache liefern (z.B. BENN et al. 2004, EvANs et al. 2006).

Die durch den Beitrag von van DER MeER et al. (2003) ausgelöste Diskussion zur Tillklassifikation ist in jüngerer Zeit durch ein neues Nomenklaturkonzept abgelöst worden: In einem umfangreichen Überblick zum Stand der Tillforschung tragen EvaNs et al. (2006) einen Großteil der verfügbaren Daten zur Till-Sedimentologie aus makro- und mikroskopischen Arbeiten, Laborversuchen und geophysikalischen Messungen an der Basis rezenter Gletscher zusammen. Evans et al. (2006) schlussfolgern, dass fast alle Tills das Produkt einer Prozesskopplung mehrerer subglazialer Abläufe sind, die sich sowohl zeitlich als auch räumlich überlagern (bezeichnet als ,polythermales-polyrheologisches subglaziales Mosaik'). Je nach den subglazialen Rahmenbedingungen tragen Ablagerung und Deformation (einschließlich der Homogenisierung präexistenter Sedimente) demnach zur Entstehung eines Tills bei, wobei der Anteil der jeweiligen Teilprozesse als variabel einzustufen ist. Entscheidend ist dabei die Erkenntnis, dass die frühere prozessspezifische Differenzierung in reine Lodgement, Deformation und Basal Meltout Tills (s. Abb. 2) der Komplexität und Überlagerung verschiedener tillgenetischer Prozesse nicht ausreichend Rechnung trägt.

Evans et al. (2006) empfehlen daher, künftig nur noch solche Tilltypen terminologisch zu unterscheiden, die als klar trennbare Einheiten beschreibbar sind und für die objektiv nachvollziehbare diagnostische Kriterien existieren. Danach wird der neu definierte ,subglaziale Traktionstill (engl. subglacial traction till) in die Nomenklatur eingeführt, der sowohl die vormaligen Typen ,Setztill' (lodgement till) und Deformationstill (deformation till) ersetzt und subsummiert als auch Sonderfälle wie Leeseitenverfüllungen (lee-side cavity fills) umfasst. Ferner schlagen Evans et al. (2006) vor, die Begriffe ,subglazialer Ausschmelztill (subglacial melt-out till) und Sublimationstill (sublimation till) vorerst beizubehalten, diese jedoch aufgrund der beschriebenen Probleme bezüglich der Genese und des Erhal- 


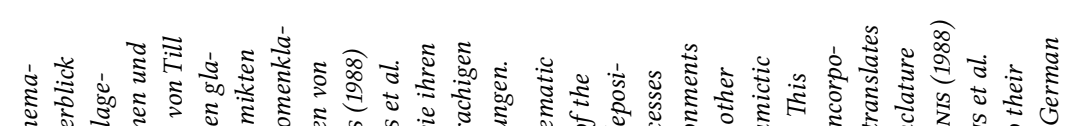

फٓ

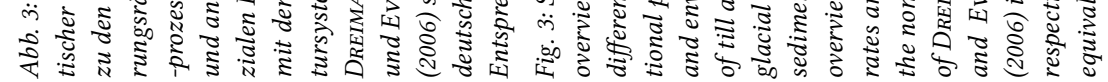

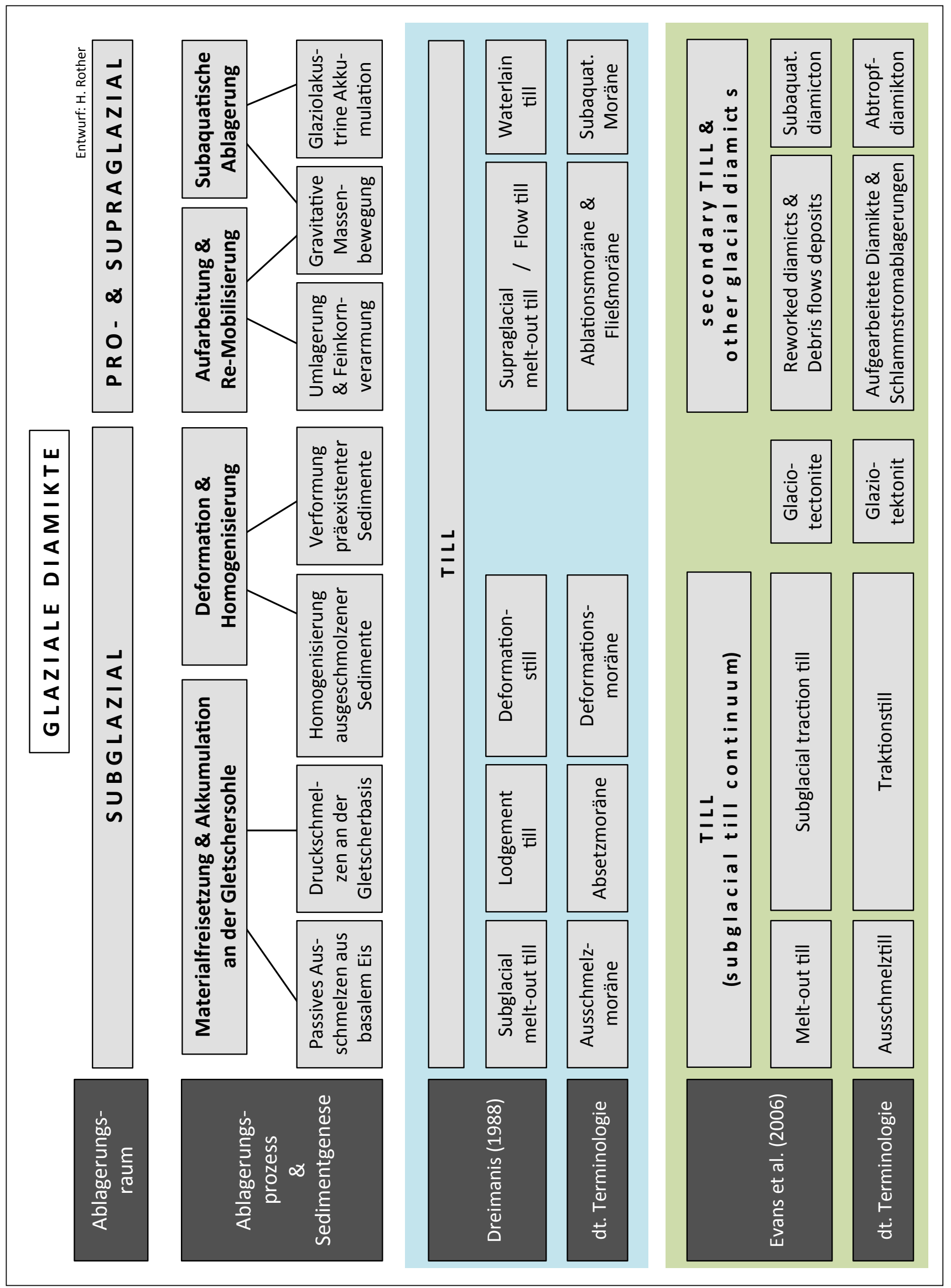


tungspotentials zunächst noch als theoretische Konzeptbegriffe zu betrachten, die weiterer rigoroser Überprüfung an rezenten Gletschern bedürfen und nicht unkritisch verwandt werden sollten. Im Folgenden werden die hier eingeführten Till-Begriffe nochmals definiert und nach ihren Haupteigenschaften beschrieben (Evans et al., 2006: 169). Eine zusammenfassende Darstellung der Ablagerungsräume, genetischen Prozesse und verschiedenen Nomenklatursysteme für Till und verwandte glaziale Diamikte findet sich in Abb. 3.

Subglazialer Traktionstill (STT; subglacial traction till) Subglaziale Sedimentablagerung eines Gletschers, der entweder aktiv über sein Bett gleitet und/oder dieses im Zuge der Bewegung durchgreifend deformiert (deformable bed). Die Freisetzung von Material an der Gletschersohle erfolgt zumeist durch Druckschmelzen, in anderen Fällen wird Material vom bestehenden Substrat losgelöst, disaggregiert und weitestgehend durch Scherung homogenisiert. Traktionstills weisen typischerweise scharfe bzw. erosive
Kontakte zu liegenden Einheiten auf, die zum Teil durch Scherung oder Schleppfalten in den STT inkorporiert werden (Abb. 4a). STTs können ein sowohl massiges als auch klüftiges Erscheinungsbild zeigen, wobei Geschiebe häufig in Eisfließrichtung eingeregelt sind (Richtungsgefüge).

\section{Subglazialer Ausschmelztill(SAT;subglacialmeltouttill)}

Durch stagnierende oder langsam fließende Gletscher gebildete subglaziale Diamikte, welche durch Materialfreisetzung aus schuttreichem basalem Eis entstehen und nicht durch nachfolgende gravitative Umlagerung, Deformation oder fluviale Auswaschung überprägt wurden. Unter $\mathrm{Zu}$ grundelegung der an rezenten Gletschern beobachteten Mächtigkeiten schuttreichen basalen Eises (typischerweise zwischen 0,5-2,5 m) mit einem Schuttgehalt von meist unter $20 \%$ ergibt sich, dass resultierende SAT-Einheiten kaum mächtiger als ca. 0,5 m sein können. Aufgrund fehlender oder stark begrenzter Eisbewegung bei der Genese von SAT werden kaum Scherklüfte angelegt, und das für den STT typische klüftige Erscheinungsbild fehlt. Als Ar-
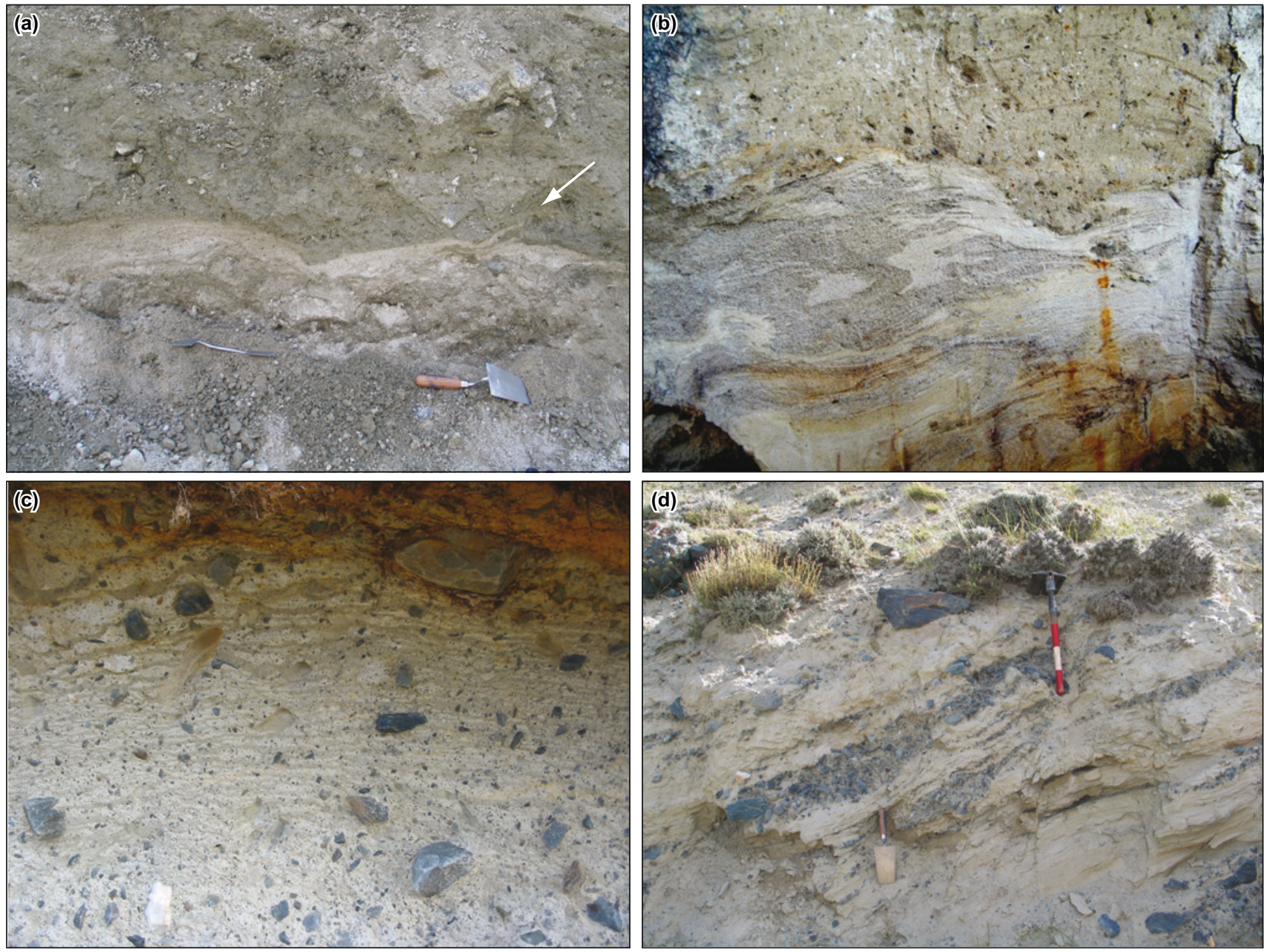

Abb: 4. (a) gefaltete Schmelzwassersande unterlagern STT, Hornkees, Österreich. (b) Typ-A Glaziotektonit aus zerscherten glaziofluviatilen Sand-und Kiesschichten, überlagert durch subglazialen Traktionstill (Glowe, Rügen); (c) Glaziotektonit (Typ A) aus stark deformierten und nahezu vollständig homogenisierten Einheiten einer ehemaligen Wechsellagerung von Schlammstromablagerungen (debris flows) und fluvialen Schichtlagen (wash horizons), Loch Shin, NW Schottland; (d) Glaziotektonit (Typ B) mit ähnlicher Wechsellagerung wie in (c), nur wesentlich geringer deformiert, so dass noch einzelne Linsen und Augenstrukturen (boudinage) klar erkennbar sind. Chagan-Tal, Russischer Altai.

Fig. 4: (a) Folded outwash underlying STT, Hornkees, Austria. (b) Type-A glaciotectonite formed from sheared glaciofluvial sand and gravel layers, overlain by STT (Glowe, Isle of Rügen); (c) glaciotectonite (type A) formed from heavily deformed and nearly completely-homogenised debris flow units and intercalated fluvial units (wash horizons), Loch Shin, NW Scotland; (d) glaciotectonite (type B) with similar alternating layers to (c), albeit much less strongly deformed, as evident from clearly-visible individual layers and augen-shaped lenses (boudinage). Chagan valley, Russian Altai. 
tefakt der direkten Ablagerung aus stratifiziertem basalen Eis weisen SATs nach manchen Autoren selbst Schichtungsmerkmale auf (HALdorsen \& SHAw 1982, Evans et al. 2006). Die obenstehend definierten Tillvarianten (STT, SAT) können nach Evans et al. (2006) als Gruppe der terrestrisch gebildeten subglazialen oder basalen Tills zusammengefasst werden.

\section{Glaziotektonit (GT; glaciotectonite)}

Bezeichnung für Lockersedimente oder brekzierte Festgesteine auch nicht-glazialen Ursprungs, welche durch subglaziale Scherung stark deformiert wurden, jedoch strukturelle Charakteristika des Ausgangsmaterials beibehalten. Je nach Intensität der tektonischen Beanspruchug (engl. stress) werden extrem stark deformierte Materialien als Glaziotektonit Typ A und solche mit geringem Verformungsgrad (engl. strain) als Typ B Glaziotektonit bezeichnet (BENN \& Evans 1996, 1998, 2010, Evans et al. 2006, Abb. 4b, c). Glaziotektonite sind nicht zu den primären Tills zu rechnen (Abb. 3, 5).

Für Till und verwandte Sedimente wurde und wird in der deutschen Terminologie häufig der zusammenfassende Oberbegriff ,Grundmoräne` genutzt, wodurch geomorphologische und sedimentologische Konnotationen vermengt werden. Problematisch ist außerdem, dass ,Grundmoränen' ein dermaßen weites Spektrum unterschiedlicher glazialer und verwandter Sedimenttypen umfassen, dass der Begriff sedimentologisch und genetisch nur von geringem Bedeutungsinhalt ist. Im internationalen Sprachgebrauch wird der Grundmoränenbegriff daher nur zur allgemeinen Beschreibung und Einordnung glazialer Landschaftsformen (z.B. hummocky ground moraine) genutzt, jedoch nicht im Sinne einer Ansprache im Rahmen der Sedimentinterpretation (z.B. Sharpe 1988, KRÜGER 1994, BENN \& EvANS 1998). Gleichlautende Empfehlungen zur Nutzung des Begriffs finden sich auch in der deutschen Literatur (LuKAs 2004, WinkLER 2009) und sollten aus Sicht der Autoren zukünftig Beachtung finden. Ein zusammenfassender Überblick zu den Merkmalen von Till und verwandten glazialen Diamikten findet sich in Abb. 5.

\subsection{Moränen: Prozesse der Landformengenese}

Trotz der zentralen Bedeutung eisrandlicher Moränen für viele Aspekte glazialgeologischer Rekonstruktion besteht auch heute noch erheblicher sedimentologischer Forschungsbedarf, um die genetischen Zusammenhänge zwischen den geomorphologischen Moränentypen und den sie aufbauenden Sedimenten systematisch $\mathrm{zu}$ verstehen (vgl. Sharp, 1984; Evans, 1989, 2009; Bennett, 2001; Evans \& Hiemstra, 2005; Lukas, 2012; Lukas et al., 2012). Wichtig ist auch hierbei, klar zwischen Form (Landform) und Inhalt (Sediment) zu unterscheiden, damit keine vorschnellen Schlüsse über die Moränengenese und deren paläoklimatische Signifikanz gezogen werden. Aus den verfügbaren Detailstudien ergeben sich drei Hauptprozesse, die an der Formung von Moränen (im geomorphologischen Sinn) maßgeblich beteiligt sind.

\subsubsection{Gravitative Prozesse}

Hierzu gehören sämtliche Prozesse, die unter Einfluss der Schwerkraft zu sedimentären Massenbewegungen und letztlich zur Bildung morphologischer Vollformen am Eisrand führen. So rutschen oder fließen auf der Eisoberfläche vorhandene Sedimente unter Schmelzwassersättigung häufig als gravitative Schlammströme ab (supraglacial debris flow, Abb. 6a), zusätzlich kommt es durch Verringerung des Porenraumes an der Oberfläche eines Schlammstroms zum Schmelzwasseraustritt und zur hangabwärtigen Verlagerung von Feinmaterial (z.B. BENN 1992, LuKAS 2005, LuKas et al. 2005, 2012, Phillips 2006, Reinardy \& LuKas 2009, Abb. 5a). Desweiteren lässt sich an vielen rezenten Eisrändern beobachten, dass supraglaziale Blöcke die Eisoberfläche herabrollen oder stürzen und in die Eisrandlage inkorporiert werden. Teilweise ergeben sich dadurch auch linienhafte Akkumulationen von Blöcken auf der Moränenoberfläche (,boulder lines') oder Blockanhäufungen zwischen Moränenfragmenten (SHARP 1984, BENN 1992, WINKLER \& Nesje 1999, Lukas 2005, 2012, Phillips 2006, ReinARdy \& LukAs 2009, Abb. 6b). Entlang eines stagnierenden, d.h. stabilen Eisrandes können diese Prozesse zur allmählichen Formation markanter Moränenrücken oder, bei sedimentären Punktquellen wie Austritten englazialer Schmelzwassertunnel, zur Bildung einzelner Moränenhügel führen (z.B. KRÜGER \& ABER 1999, LuKAS \& SASS 2011, LuKAS 2012).

In vielen Fällen werden die Materialien zunächst im direkten Kontakt zum aktiven Gletschereis oder Toteis akkumuliert, wobei letztere als Widerlager dienen und größere Aufschüttungen erst ermöglichen (LuKAs 2005, BENN \& Evans 2010). Bei nachfolgendem Rückzug verliert die Aufschüttung meist ihr Widerlager und die Form kollabiert, so dass der eis-proximale Hang der Moräne normalerweise den kritischen Böschungswinkel des Materials (je nach Steinanteil 30-35 ) erreicht (BENN 1992, LukAs 2005, 2012, LukAs et al. 2012, Abb. 6c).

Die aus diesen Prozessen resultierende Endmoränenmorphologie weist häufig eine deutliche Asymmetrie auf, die durch einen steilen eiszugewandten und einen deutlich flacheren eisabgewandten Hang gekennzeichnet ist (Abb. 5c). Letzterer repräsentiert in vielen Fällen die ehemalige Rampe eines Eiskontakt-Schuttfächers, der aus gestapelten Schlammstromablagerungen aufgebaut ist (LAWSON 1981, 1982, BENN 1992, LUKAS 2003, 2005, 2007, 2012). Im Deutschen ist für diesen Moränentyp der Begriff ,Satzendmoräne' gebräuchlich (z.B. GRIPP 1975, LIEDTKE 1981), allerdings wird diese Bezeichnung häufig aufgrund rein morphologischer Kriterien verwendet, ohne dass sedimentologische Daten zum Prozess der Moränenbildung hinzugezogen werden. Die Autoren empfehlen, auf diese genetische Interpretation zu verzichten, solange keine klaren sedimentologischen Befunde für eine Moränenbildung durch dominierend gravitative Akkumulation vorliegen.

\subsubsection{Glaziotektonisch stauchende Prozesse}

Im Gegensatz zur Satzendmoränenbildung entlang positionsstabiler Gletscher, die sich annähernd im Massengleichgewicht befinden, führen aktiv vorstoßende bzw. stark oszillierende Gletscherränder zur Bildung glaziotektonisch ge- 


\begin{tabular}{|c|c|c|c|c|}
\hline Klasse & Sedimenttyp & Untertyp & \multicolumn{2}{|c|}{ Diagnostische Merkmale } \\
\hline Primäre & \multirow{2}{*}{ Till } & $\begin{array}{l}\text { Subglazialer } \\
\text { Traktionstill }\end{array}$ & $\begin{array}{l}\text { - ungeschichtetes Sediment in bindiger } \\
\text { stark konsolidierter Grundmasse } \\
\text { - schlecht sortiert mit variablen Geschiebe- } \\
\text { anteilen in der Kies- bis Blockfraktion } \\
\text { - basaler Kontakt scharf \& oft mit Scherzone }\end{array}$ & $\begin{array}{l}\text { - massiges Erscheinungsbild oft mit aus- } \\
\text { geprägtem Kluftgefüge } \\
\text { - Geschiebe hăufig kantengerundet und ein- } \\
\text { geregelt; gekritzte \& polierte Oberflächen } \\
\text { - lokal: stark deformierte Kies- \& Sandinsen }\end{array}$ \\
\hline Diamikte & & $\begin{array}{l}\text { Subglazialer } \\
\text { Meltout Till }\end{array}$ & $\begin{array}{l}\text { - diamiktes Gemisch in sandig-schluffiger, } \\
\text { teils toniger Matrix } \\
\text { - Auftreten von Schichtungsmerkmale, Drap- } \\
\text { ierung größerer Klasten durch Feinkornlagen }\end{array}$ & $\begin{array}{l}\text { - schwächere Kompaktierung, Kluftgefüge \& } \\
\text { Scherzonen fehlen typischerweise } \\
\text { - lokal: geschichtete Kies- und Sandlinsen als } \\
\text { Reste en- \& subglazialer Schüttungen }\end{array}$ \\
\hline \multirow{4}{*}{ Sekundäre } & $\begin{array}{l}\text { subglazial defor- } \\
\text { mierte Locker- \& } \\
\text { Festgesteine }\end{array}$ & Glaziotektonit & $\begin{array}{l}\text { - präexistierende durch den Gletscher sub- } \\
\text { glazial deformierte Materialien } \\
\text { - Glaziotektonite enthalten lithologische \& struk- } \\
\text { turelle Merkmale des Ausgangsgesteins }\end{array}$ & $\begin{array}{l}\text { - Typ A: intensiv geschert und brekziiert; prä- } \\
\text { existente Merkmale nur undeutlich erhalten } \\
\text { - Typ B: niedriggradig und va. duktil deformiert; } \\
\text { präexistete Merkmale deutlich erhalten }\end{array}$ \\
\hline & $\begin{array}{l}\text { Gravitative } \\
\text { Massen- } \\
\text { bewegungen }\end{array}$ & $\begin{array}{l}\text { Schlammstrom- } \\
\text { ablagerung }\end{array}$ & $\begin{array}{l}\text { - durch Rutschung oder Abfließen von der Eis- } \\
\text { oberfläche mobilisierter glazialer Schutt } \\
\text { - häufig grob stratifiziert, teilweise gradiert }\end{array}$ & $\begin{array}{l}\text { - Auftreten von charakteristischen Fließstruk- } \\
\text { turen sowie Rutsch- und Schleppfalten } \\
\text { - am Schichtkontakt zu unterlagernden Ein- } \\
\text { heiten oft Belastungsmarken }\end{array}$ \\
\hline & $\begin{array}{l}\text { Sekundär } \\
\text { aufgearbeitete } \\
\text { Diamikte }\end{array}$ & $\begin{array}{l}\text { Ablations- } \\
\text { diamikton }\end{array}$ & $\begin{array}{l}\text { - im Niedertauprozess gebildetes Diamikton } \\
\text { mit zahlreichen supraglazialen Komponenten } \\
\text { - meist sandige Matrix; vermutlich infolge von } \\
\text { sekundärer Durchspullung an Feinkorn verarmt }\end{array}$ & $\begin{array}{l}\text { - teilweise schwache Schichtungsmerkmale } \\
\text { und Hinweise auf gravitative Umlagerung } \\
\text { - vergleichsweise schwach kompaktiert }\end{array}$ \\
\hline & $\begin{array}{l}\text { Subaqua- } \\
\text { tische } \\
\text { Diamikte }\end{array}$ & $\begin{array}{l}\text { Abtropf- } \\
\text { diamikton }\end{array}$ & $\begin{array}{l}\text { - sub- oder proglazial gebildetes geschiebe- } \\
\text { reiches glaziolakustrines Diamikton } \\
\text { - häufig im Verbund mit feinkörnigen, geschich- } \\
\text { teten teilweise auch laminierten Sedimenten }\end{array}$ & $\begin{array}{l}\text { - Bildung als subaquatischer Schlammstrom } \\
\text { oder durch das Abtropfen von Material aus } \\
\text { einer aufschwimmenden Gletscherbasis } \\
\text { - synsedimentär \& glaziotektonisch deformiert }\end{array}$ \\
\hline
\end{tabular}

Abb. 5: Zusammenstellung wichtiger Merkmale für die Geländeansprache und Klassifikation verschiedener Tilltypen und verwandter glazialer Diamikte.

Fig. 5: Compilation of key criteria for the description, interpretation and classification of different till types and associated glaciogenic diamictic sediments in the field.

prägter Stauchendmoränen. In Abhängigkeit von der Höhe und Steilheit des Eisrandes, sowie der Vorstoßdauer und der Beschaffenheit des vor dem Gletscher liegenden Materials, können Stauchendmoränen (engl. push moraines) beachtliche Ausmaße annehmen. Durch eine Kombination von Auflast und Vorwärtsbewegung überträgt der Gletscher erheblichen kompressiven Druck in das Vorland und verursacht die Auffaltung und Verschuppung proglazialer Sedimentpakete (Evans 1989, BenN \& Evans 1993, LuKas 2005, 2007, 2012, s.u. Abb. 7). Daneben können auch bereits vorhandene Moränen anderen Typs durch spätere Vorstöße strukturell überprägt und zu Stauchendmoränen umgebildet werden (LuKAs 2005, 2007, BenN \& Lukas 2006, BENN \& Evans 2010). Räumlich gestaffelte Moränenrücken, meist mit einem Abstand von einigen hundert Metern zwischen den einzelnen Kämmen, entstehen entweder im Zusammenhang mit mehreren diskreten Eisvorstößen oder einer Staffelung von Moränenpositionen im Kontext einer rezessiven Gletscherentwicklung.

Große glaziotektonische Stauchkomplexe bilden in vielen Räumen die eindruckvollsten Moränen, die als Zeugnisse der pleistozänen Vereisungen erhalten geblieben sind. Hinsichtlich der Genese sehr großer und breiter Moränenzonen, die meist aus mehreren hintereinandergeschalteten Rücken bestehen, legen Beobachtungen in rezenten Analogsituationen (z.B. Spitzbergen) eine Bildung im Zusammenhang mit schnell vorstoßenden Gletschern (sog. surging glaciers) nahe (Lovell 2014, Lovell et al. 2015). Die komplex aufgebauten Block-Stauchkörper (engl. thrust block moraines), die beispielhaft im Kreide-Stauch- komplex Jasmund auf Rügen ausgebildet sind, bestehen meist aus einer großdimensionalen Sattel- und Muldenstruktur mit vielen Lagerungsstörungen, die als proglaziale Aufpressung von Stauchwällen angelegt und im Zuge fortschreitender Raumeinengung in eine glaziotektonische Schuppenstruktur weiterentwickelt werden. Auch die Moräne der Dammer Berge wird von VAN DER WATEREN (1995) aufgrund aufgeschlossener gefalteter Schuppenstrukturen aus proglazial abgelagerten Schmelzwassersedimenten als ein glaziotektonischer Stauchkomplex (thrust block moraine) interpretiert. Die Sedimente, die diesen Moränenbogen aufbauen, deuten dabei darauf hin, dass ein aus Norden vorstoßender Lobus des Nordischen Inlandeises hauptsächlich bereits vorhandene proglaziale Sedimente zusammenstauchte, währenddessen eigene subglaziale Sedimente kaum zur Ablagerung kamen. Im Einzelfall sind großräumige Areale, welche morphologisch einen Stauchmoränencharakter aufweisen, nur durch detaillierte sedimentologische und strukturgeologische Aufnahmen als Stauchendmoränen oder komplexe Stauchkörper (thrust block moraines) zu interpretieren, die ihrerseits häufig eng mit surging glaciers, also extrem schnell vorstoßenden Gletschern, in Verbindung gebracht werden (BENN \& EvANs 2010).

\subsubsection{Basales Anfrieren und Verfrachten von Sedimentpaketen}

Allgemein dauert es mehrere Jahre (z.T. Jahrzehnte), bis sich klimatische Veränderungen ausreichend auf die Mas- 

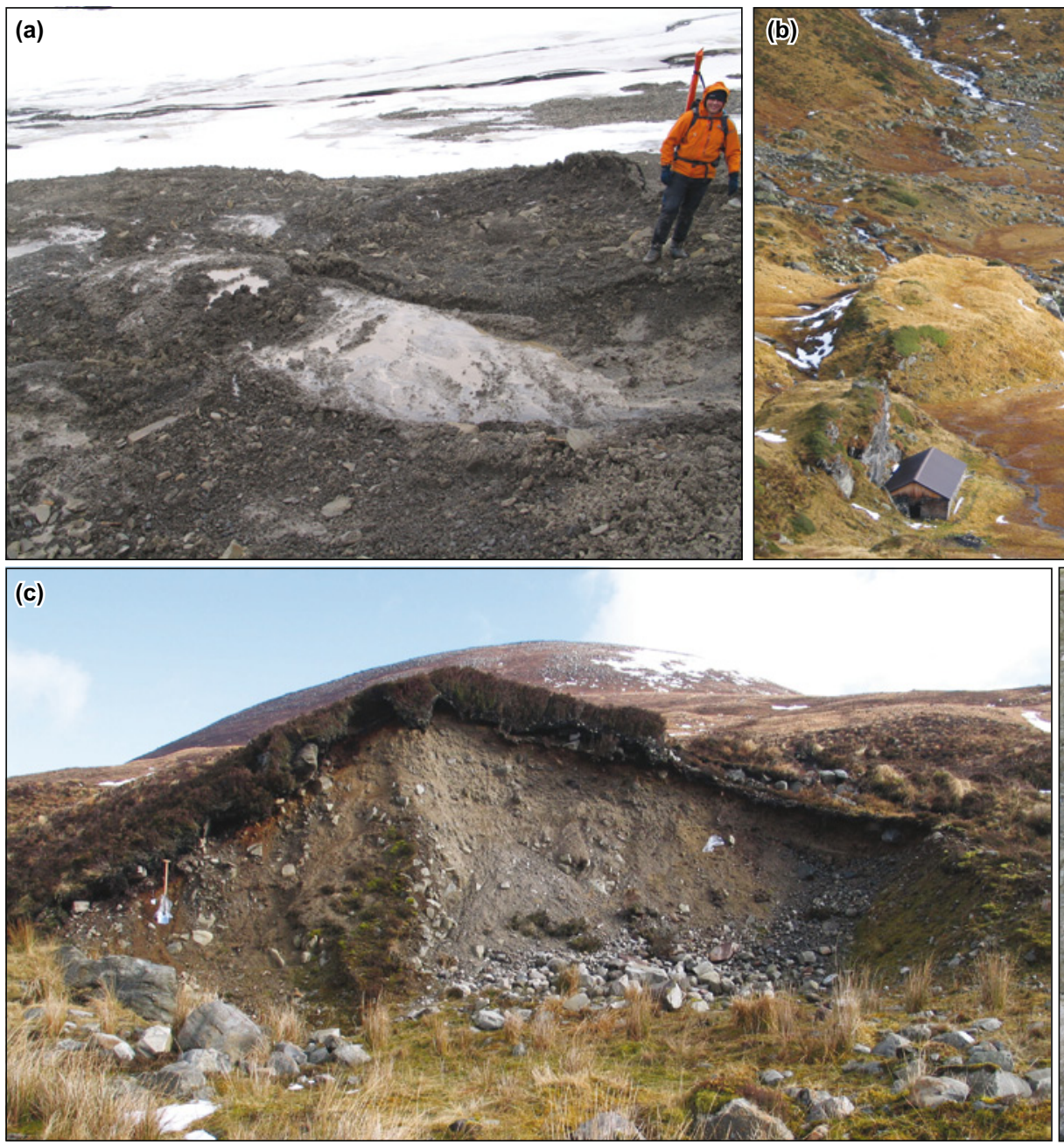
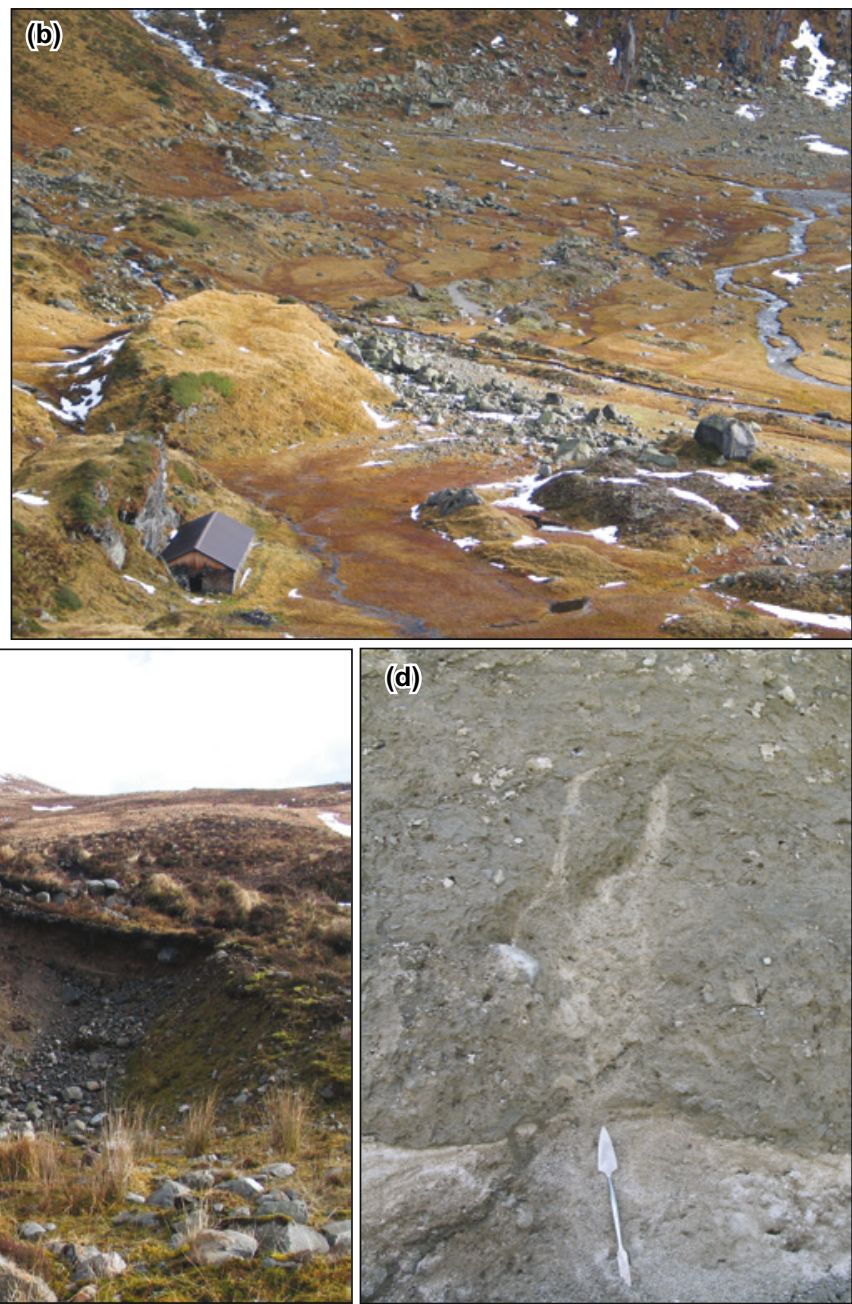

Abb. 6: (a) Aufnahme eines langsam-fließenden supraglazialen Schlammstromes (debris flows) mit diamiktischem Lobus (links) und feinkörnigem Aufschwemmsediment (wash horizon); Nordenskiöldtoppenbreen, Svalbard. (b) Moränenrücken, der aus diamiktischen und bewachsenen Rückenfragmenten und Blocklinien (boulder lines) besteht. Einige der Blöcke erreichen die Höhe des $7 \mathrm{~m}$ hohen Schuppens im linken Vordergrund und belegen, dass es sich hierbei um eine Satzmoräne an einem stationären Eisrand (dumping) anstelle von einer Stauchmoräne an einem vorstoßenden Eisrand handelt. Kleineiszeitliche Maximalausdehnung, Schwarzensteinkees, Österreich. (c) Asymmetrische Satzmoräne in NW Schottland mit proximalem Hang links. Sedimentologisch handelt es sich hierbei um einen Eiskontaktfächer, dessen Oberfläche nach rechts seicht einfällt und dessen proximaler Hang aus Material im kritischen Böschungswinkel aufgebaut ist, welches beim Eisrückzug durch Kollabieren entstanden ist (s. LUKAS 2005). (d) Injektion basaler Sande und Silte (water escape structures) in umlagernden subglazialen Traktionstill; diese wurden während und nach der Injektion in diesem Fall im Hangenden durch Gletscherbewegung nach rechts verschleppt; Hornkees, Österreich.

Fig. 6: (a) Slowly-moving supraglacial debris flow showing the wrinkled diamictic lobe that forms due to flow compression and dewatering (on the left) and the fine-grained wash horizon to its right; Nordenskiöldtoppenbreen, Svalbard. (b) moraine ridge consisting of diamictic and vegetated ridge fragments and boulder lines. Some boulders reach the heights of the $7 \mathrm{~m}$-high shed in the left foreground and provide tentative evidence that this moraine was formed primarily by dumping rather than bulldozing. Little-Ice Age maximum, Schwarzensteinkees, Austria. (c) asymmetric dump moraine in NW Scotland. Sedimentologically, this moraine represents a terrestrial ice-contact fan, with the fan surface sloping gently to the right. The proximal slope on the left repesents an ice-contact slope with material at the angle of repose, formed when the proximal part of this moraine lost its support due to ice retreat (cf. LuKAS 2005). (d) injection of basal silts and sands (water escape structures), which were deformed to the right during overriding post-injection; Hornkees, Austria.

senbilanz eines Gletschers auswirken, um zu einer veränderten Dynamik der Gletscherfront (Vorstoß, Rückzug, Stagnation) zu führen. In selteneren Fällen reagieren Eisränder auch auf saisonale Schwankungen der Lufttemperatur, wobei sich kleinförmige jahreszeitliche Moränen bei Wintervorstößen ausbilden (KRÜGER 1994, 1995, LUKAS 2012, ReINARDy et al. 2013, BRADWELl et al. 2013, CHANDLER et al. 2016; siehe auch Abschnitt 4.2). Für die diesbezügliche Moränengenese ergeben sich besondere Faktoren, da es sich typischerweise um das räumlich begrenzte Vorstoßen (bzw. Oszillieren) geringmächtiger Eisränder handelt. Unter diesen Rahmenbedingungen kann es, bei starker Ausbildung winterlichen Bodenfrosts, zum kurz- zeitigen Anfrieren ausgedehnter subglazialer Sedimentpakete an die Gletschersohle kommen, die dann im Zuge des winterlichen Eisvorstoßes über kurze und mittlere Distanzen verschleppt werden (basal freeze-on and transport of sediment slabs). Schmilzt der Eisrand dann im Frühjahr zurück, verbleiben die bis zu ca. 1,5 m mächtigen Sedimentlagen innerhalb der relativ kleinen, saisonal gebildeten Stauchmoränen erhalten. Bei sich wiederholenden intensiven saisonalen Eisfrontoszillationen kann dieser Prozess zur Aufstapelung und folgender Aufstauchung mächtiger Pakete subglazialen Materials führen, wie sie an rezenten Gletschern in Island (KRÜGER 1994, 1995, EvANS \& Hiemstra 2005, Bradwell et al. 2013, Chandler et al. 
2016) Nordnorwegen (MATthews et al. 1995, HiEmstra et al. 2015) und den europäischen Alpen beobachtet wurden (LuKAs 2012). Ein wichtiges Fazit bei der Betrachtung moränengenetischer Prozesse ist, dass die o.g. Prozesse nur äußerst selten singulär auftreten - eine reine Satzendmoräne ist ebenso selten wie eine reine Stauchendmoräne! Gleiches gilt auch für Moränen, die durch basal angefrorene Sedimentpakete aufgebaut werden: auch hier können sowohl gravitative Umlagerungsprozesse als auch glaziotektonische Stauchung das sedimentologisch-geomorphologische Endprodukt maßgeblich mitprägen. Zusammenfassend soll unterstrichen werden, dass die meisten Moränen genetisch auf eine Kombination verschiedener Prozesse zurückgehen und somit Mischformen darstellen, wobei die endgültige Klassifikation einer Moräne nach der dominanten Komponente vorgenommen werden sollte. Vor allem bei größeren Moränen (z.B. Lateralmoränen, Moränenkomplexen) ist eine genaue und möglichst ausführliche Sedimentaufnahme wichtig, um u.a. grobe Fehleinschätzungen, was glaziodynamische oder paläoklimatische Schlußfolgerungen anbelangt, auszuschließen. Nur aufgrund der Sedimentaufnahme kann hier z.B. festgestellt werden, ob ein Moränenrücken tatsächlich - wie zumeist angenommen - einer einzelnen Oszillation bzw. einem Vorstoß entspricht, oder ob darin mehrere Vorstöße repräsentiert sind, die durch erosive Kontakte, abgelagerte Zwischensedimente oder Paläoböden angezeigt werden (LukAs et al. 2012).

\section{Diskussion}

\subsection{Moräne versus Till}

Ältere Darstellungen der Glazialen Serie zeigen oft einen engen schematischen Zusammenhang zwischen subglazialem Till und den Sedimenten, welche die (End-)Moränen direkt aufbauen (siehe z.B. Abbildungen in LouIs $\mho$ Fischer 1979, LiedtKe 1981, Zepp 2002). Aus den oben dargelegten Beobachtungen zur sedimentären Architektur von Endmoränen geht jedoch hervor, dass in der Realität meist keine direkte Verzahnung von Till und den eigentlichen Moränensedimenten zu finden ist. Die Sichtung der verfügbaren Literatur zeigt, dass die meisten Endmoränen aus Lithofaziesassoziationen bestehen, welche sich grob in Sand, Kies und diamiktische Einheiten unterteilen lassen. Die sedimentären Merkmale der diamiktischen Einheiten legen nahe, dass es sich hierbei nicht um primär glaziogene (direkte Ablagerung vom Eis), sondern weit häufiger um gravitativ verlagerte Sedimente (Massenbewegungen) handelt. Daher sollten diese Sedimente auch nicht als Till (sensu stricto) angesprochen werden. Aufgrund der sedimentologischen Vielfalt gibt es kein „klassisches“ Moränensediment, insofern ist der häufig gebrauchte Begriff „Moränenmaterial“ zur allgemeinen Beschreibung des Sedimentinventars von Moränen durchaus treffend unscharf, jedoch sollte dieser Begriff nicht als Synonym für ,Till' verwendet oder verstanden werden.

In der Literatur gibt es selten genauere Angaben zu den Mengenverhältnissen der verschiedenen Moränensedimente, was v.a. in der häufig schlechten Aufschlusssituation der meisten Endmoränen begründet liegt: Moränen aus groben diamiktischen Materialien zu durchgraben ist ein schwieriges Unterfangen wie zahlreiche Feldstudien belegen (z.B. Pindur \& Heuberger 2008, Lukas \& Sass 2011, Lukas 2012, Lukas et al. 2012). Hinzu kommt, dass sich Moränen im Querschnitt zur Basis stark verbreitern, und sich mit zunehmender Tiefe das zu evakuierende Materialvolumen stark erhöht, so dass ein Aufgraben der tatsächlichen Moränenbasis in den meisten Fällen unterbleibt. In Fällen, wo dies dennoch gelang (z.B. BENN 1990), ließen sich ausgedehnte Lagerungsstörungen im Kontakt zu den liegenden Sedimenten dokumentieren, die auf den Einfluss glaziotektonischer Aufarbeitung und Einschuppung der liegenden Vorlandsedimente bei der Moränengenese hindeuten (Abb. 7). Zungenartig entwickelte vertikale Einschaltungen von Teilen der basalen Lithofazies in die hangenden Einheiten der Moräne entstehen dabei häufig als Entwässerungsstrukturen, die während des Vorstoßes durch Wasserüberdruck zur Injektion basaler Sedimente in darüber lagernde Einheiten führen (water escape structures; Lowe 1975, vAN DER MEER et al. 2001, BENN \& EvANs 2010, Abb. 6d). Die Moränen selbst bestehen häufig aus gefalteten Sanden, Kiesen und vereinzelten Diamikten, wobei die typischen Störungen entweder das Resultat kompressiver glaziotektonischer Prozesse sind (Aufschiebungen, überkippte Falten etc.), oder durch Kollapsprozesse (Abschiebungen, Rutschfalten etc.) entstanden sind.

\subsection{Empfehlungen für die Beschreibung und Interpretation glazialer Sedimente}

Grundsätzlich ist bei der sedimentologischen Aufnahme glazialer Sedimente darauf zu achten, dass der zu bearbeitende Aufschluss nicht zu klein bemessen ist, da die Erfahrung zeigt, dass konkrete sedimentgenetische Deutungen auf Grundlage zu kleiner Aufschlüsse meist nicht verlässlich sind. Als Faustregel gilt der Grundsatz ,je detaillierter die geologische Fragestellung, desto größer der nötige Aufschluss“. Vor Beginn jeder Aufnahme ist die zu bearbeitende Profilwand soweit zu säubern bzw. zurückzulegen, dass frische Aufschlussverhältnisse zur Verfügung stehen. Im ersten Arbeitsschritt erfolgt eine grobe lithofazielle Einteilung der vorliegenden Einheiten, wobei u.a. zunächst nach diamiktischen und besser sortierten groboder feinklastischen Fazies unterschieden werden kann. Es folgt die einheitenbezogene Beschreibung sedimentärer und struktureller Merkmale, die den allgemeinen Richtlinien zur Aufnahme klastischer Sedimentgesteine folgt und dabei alle wichtigen Gefügekriterien (wie Korngröße, Sortierung, Schichtungsmerkmale etc.) genauso berücksichtigt wie die Beschreibung der Lagerungsverhältnisse (Schichtneigung, Schichtgeometrie, Art der Schichtkontakte) und möglicherweise auftretende Deformationsstrukturen (siehe ausführliche Anleitungen zur Aufnahme klastischer Sedimente in Evans \& BENN 2004b sowie in STOW 2008). Zusätzlich sollten für jede diamiktische Einheit Angaben zum Verfestigungsgrad (Kompaktion), möglicherweise auftretenden Einregelungsgefügen (z.B. Längsachseneinregelung) (BENN 2004a) und der Geschiebeform (Häufigkeit von geschossähnlichen Klastgeometrien, Abschätzung des Rundungsgrades, Auftreten von Kritzungen und Polierung der Geschiebeoberflächen) erfolgen (BENN 2004b, LuKAS et al. 2013). Um die Aufnahme möglichst systematisch und 



Abb. 7: (a) Photo und (b) 2-dimensionale Detailaufnahme des Aufschlusses GOR 5 (s. LUKAS 2012) einer jahreszeitlichen Stauchmoräne am Gornergletscher, Schweiz. Der eiszugewandte (proximale) Hang ist links. Schwarz: Blöcke; ockerfarben: Sand- und Siltlagen; gestrichelte Linien deuten Schichtung (schwarz) bzw. schwächere Stratifizierung der Diamikte (blau) an. Lithofacies-codes nach EVANS \& BENN (2004). Die aufgescherte Faltenstruktur ist deutlich zu erkennen; die Faltenachse ist parallel zur Kammlinie der Moräne, was strukturgeologisch deren Genese durch den Eisvorsto $\beta$ bestätigt.

Fig. 7: (a) photo and (b) 2-dimensional log of exposure GOR 5 (cf. LUKAS 2012) through an annual moraine formed by Gornergletscher, Switzerland. The proximal slope is on the left. Black envelopes represent boulders, ocrecoloured areas sand and silt layers; dashed black lines indicate bedding while blue dashed lines indicate weaker stratification within diamictic units. Lithofacies codes follow EVANS \& BENN (2004). The bulldozed and sheared overturned fold is clearly visible; its fold axis is parallel to the crestline of this moraine, which demonstrates genesis of this moraine by push during a winter readvance. zeitlich effizient zu gestalten, eignet sich der Einsatz von Lithofazies-Kodierungen zur Beschreibung der Einheiten (z.B. nach Eyles et al. 1983, KRÜGER \& KJÆR 1999, Evans \& BENN 2004b) sowie die Nutzung von Fazies-Aufnahmeblättern (siehe KRÜGER \& KJÆR 1999). Um die Aufschlussinterpretation für Dritte nachvollziehbar $\mathrm{zu}$ gestalten, ist es unerlässlich, den Aufschluss durch Skizzen und Fotos zu dokumentieren. Hierbei ist anzumerken, dass die alleinige Dokumentation durch Fotos nicht ausreichend detailliert ist. Am effizientesten ist in diesem Zusammenhang die Anlage einer maßstäblichen Zeichnung, die im Detailgrad der Realität so weit wie möglich entspricht. Gerade in Fällen, in denen ein Aufschluss nur einmalig zugängig ist, wie im Rahmen einer Feldkampagne in entlegenen Gegenden, ist diese Methode einem Fotomosaik und einigen wenigen Skizzen vorzuziehen, da mit dem Auge im Gelände gemachte Beobachtungen auf Fotos später selten nachvollzogen werden können.

\subsection{Zusammenfassung: Praktische Hinweise für die Ansprache glazialer Diamikte und glazialer Landformen}

Wie bei jeder sedimentologischen Aufnahme gilt es, möglichst viele Daten bereits im Gelände systematisch und präzise aufzunehmen und diese entsprechend detailliert zu dokumentieren. Wie in diesem Beitrag dargelegt, sollte zukünftig für Sedimente, die aus einem Gemisch verschiedener Korngrößen bestehen, bei der Beschreibung der deskriptive und genetisch neutrale Begriff ,Diamikton'verwendet werden. Eine Interpretation als Till ist erst dann gesichert, wenn die entsprechenden Kriterien, die auf eine subglaziale Genese hindeuten, erfüllt sind. Das unreflektierte Ersetzen der für glaziogene Sedimente allgemein verwandten deutschen Begriffe durch die Bezeichnung ,Till ist ohne eingängige Beschreibung und Begründung dieser Interpretation nicht mehr adäquat. Diese Herangehenswei- 
se entspricht der in der Sedimentologie allgemein üblichen Trennung zwischen lithofaziellen Einheiten und Fazieszuordnungen, die bereits eine genetische Konnotation tragen (z.B. Till). Mit der Nutzung des rein lithologischen Begriffs Diamikton beugt der Anwender einer vorschnellen genetischen (Fehl-) Deutung vor, da zu beachten ist, dass diamiktische Sedimente nicht nur durch subglaziale tillbildende Prozesse entstehen, sondern ebenso häufig durch supraoder proglaziale Vorgänge (z.B. Schlammströme) sowie eine Reihe nicht-glazialer Prozesse gebildet werden können. Auch innerhalb glazialer Sedimentabfolgen ist nicht davon auszugehen, dass die dortigen Diamikte gänzlich oder auch nur zum überwiegenden Teil als Till anzusprechen sind (vgl. Krienke 2003, Ludwig 2005, Rother et al. 2010, Evans et al. 2013).

Wie in diesem Beitrag dargelegt, sollte der Begriff ,Moräne' zukünftig ausschließlich im geomorphologischen Sinne und damit für die Beschreibung glazialer Landformen verwendet werden. Vor jeder genetischen Interpretation ist es erforderlich, die betreffende Landform (bzw. Landformvergesellschaftung) nach morphologischen Kriterien detailliert zu kartieren (Verbreitung, Größe, Orientierung, Hangneigung etc). In Fällen, in denen dafür sehr exakte Daten erforderlich sind (z.B. Differenzierung von Strandterrassen), sollten diese Parameter mit einem Theodoliten oder D-GPS vermessen werden. In Abhängigkeit von der Fragestellung der glazialen Untersuchung kann in geeigneten Fällen bereits ein klarer Oberflächenbefund genügen (z.B. Kartierung von Endmoränenpositionen für Rekonstruktion der Maximalausdehnung einer Vereisung). Wenn es jedoch um weiterführende genetische Interpretationen geomorphologischer Elemente geht, sollten diese stets durch sedimentologische Aufnahmen abgesichert werden. Wo dies aufgrund fehlender Aufschlüsse nicht adäquat möglich ist, sollte die gezielte Anlage von tieferen Schürfen erwogen werden. Sollte auch dies nicht erreichbar sein, kann der Einsatz geophysikalischer Prospektionsmethoden, wie des Georadars (ground-penetrating radar; GPR) in Erwägung gezogen werden. Einschränkend ist jedoch darauf hinzuweisen, dass die Ausweisung und Interpretation verschiedener Radarfazies ohne die testweise Korrelation zu Bohrungen oder Aufschlüssen gerade bei hochvariablen glazialen Sedimentabfolgen häufig problematisch ist (s. LuKAS \& SASS 2011 und darin zitierte Arbeiten). Eine kombinierte Herangehensweise basierend auf geomorphologischen und sedimentologischen Methoden ist daher solchen Ansätzen stets vorzuziehen.

Da glaziale Ablagerungen und Landschaftsformen zusätzlich wichtige Informationen zur Dynamik ehemaliger Vergletscherungen sowie der paläoklimatischen Entwicklung liefern können, ist deren absolute geochronologische Datierung (mittels ${ }^{14} \mathrm{C}$-Methode, Optisch Stimulierter Lumineszenz oder durch kosmogene Nuklide) in vielen Situationen von großem Interesse. Um hierbei zu verlässlichen Ergebnissen zu kommen, ist die korrekte genetische Ansprache der zu datierenden Materialien von entscheidender Bedeutung. Besonders wichtig ist dabei zu klären, in welchem Bezug die beprobten Materialien zum ehemaligen Gletscher standen und ob es sich um subglaziale, proglaziale oder paraglaziale Ablagerungen handelt. Insbesondere ist zu berücksichtigen, dass in vielen Fällen das da- tierte Material nicht direkt aus den glaziogenen Ablagerungen selbst, sondern aus stratigraphisch unterlagernden (eisüberfahrenen) oder überlagernden Schichten (z.B. Löss, Paläoböden) stammt, bzw. aus eisrandnahen Ablagerungen (z.B. fluviale oder limnische Sedimente) gewonnen wurde. Da die genaue zeitliche Differenz zwischen Ablagerung der datierten Materialien und dem Gletschervorstoß zumeist unbekannt ist, ergibt sich die Einschränkung, dass viele der resultierenden Altersbestimmungen (je nach stratigraphischer Position der datierten Materialien) nur als Maximaloder Minimalalter zu werten sind. Sollten die datierten Moränensedimente zusätzlich Anzeichen für ein nochmaliges Überfahren durch den Gletscher zeigen, kommt hinzu, dass solche Moränen weder zuverlässige Anzeiger für die maximale Eisausdehnung sind, noch würde die Datierung den Zeitpunkt des letzten glazialen Events im untersuchten Raum erfassen.

Hinsichtlich der Optisch Stimulierten Lumineszenzdatierung (OSL) zur Altersbestimmung glazialer Ablagerungen ist anzumerken, dass sich der Anwender über die Genese dieser Sedimente bewusst sein muss, um eine Fehlinterpretation der Datierungen ausschließen zu können. Glaziale Sedimente im Allgemeinen und diamiktische Einheiten im Besonderen haben aufgrund der Sedimentationsbedingungen meist nur geringe Aussichten auf eine vollständige Signalbleichung vor der Ablagerung. Hinzu kommen bei intra-diamiktischen Sandlinsen bzw. geringmächtigen interdiamiktischen Sandeinschaltungen dosimetrische Unsicherheiten, weshalb diese für OSL-Datierungen nur unter Vorbehalten geeignet sind (AITKEN 1998, Preusser et al. 2008).

Durch die Entwicklung der Oberflächenexpositionsdatierung mittels kosmogener Radionuklide $\left({ }^{10} \mathrm{Be},{ }^{26} \mathrm{Al},{ }^{36} \mathrm{Cl}\right)$ haben sich in jüngerer Zeit neue Möglichkeiten der direkten Datierung von Moränen ergeben. Neben einer Beachtung der stringenten Qualitätskriterien für die Auswahl geeigneter Proben sollte sich der Anwender bewusst sein, dass die resultierende Datierung nur die letzte Phase der Oberflächenbildung der Moräne, d.h. kurz vor Aufgabe der zu datierenden Eisposition durch Eisrückzug, zeitlich erfasst. Da in vielen Fällen austauendes Toteis zu einer zeitlichen Verzögerung in der Oberflächenstabilisierung der Moräne führt, sind Expositionsdatierungen durch kosmogene Nuklide streng genommen zunächst als Minimumalter aufzufassen (vgl. LüTHGENs \& BösE 2012). Fallstudien an großen Seitenmoränen alpiner Vereisungsgebiete zeigen zudem, dass diese Moränen oft das Resultat mehrerer glazialer Ereignisse sind, und häufig während mehrerer aufeinanderfolgender Eisvorstöße überschüttet wurden und damit mehrphasige Bildungen darstellen (s. KIRKBRIDE \& WinkLer 2012, LukAs et al. 2012). Für die Oberflächendatierung von Moränen bedeutet dies, dass meist nur die letztgebildete Oberfläche, und damit das jüngste Ereignis, zeitlich erfasst wird.

\section{Danksagung}

Wir möchten uns bei allen Kollegen und Kolleginnen sowie den im Rahmen unserer Forschungsarbeiten betreuten Studenten bedanken, die durch Diskussionen, Hilfe bei Grabungen im Gelände und kritische Anmerkungen diesen Bei- 
trag beeinflußt haben. Stellvertretend seien hier genannt: Doug Benn, Clare Boston, Ben Chandler, Dave Evans, Ole Humlum, Heiko Hüneke, Susan Ivy-Ochs, Hanns Kerschner, Harold Lovell, Benny Reinardy, Christian Schlüchter, Jamie Shulmeister, Jaap van der Meer und Dirk van Husen. Den Teilnehmern der DEUQUA Jahrestagung in Innsbruck im September 2014, besonders Frank Preusser, Daniela Sauer und Holger Freund, sei für den Gedankenanstoß zu diesem Artikel gedankt. Wir danken Christopher Lüthgens und Stefan Wansa für die konstruktiven Gutachten.

\section{Literatur}

AgAssiz, L. (1838): Des glaciers, des moraines et des blocs eratiques. - Ac tes Societas Helvetiae Scientis Naturae, 22: 5-32.

Aitken, M.J. (1998): An introduction to optical dating. - 267 S., Oxford (Oxford Science Publications)

BAnHAM, P.H. (1977): Glacitectonites in till stratigraphy. - Boreas, 6: 101-105.

BELL, F.G. (2002): The geotechnical properties of some till deposits occurring along the coastal areas of eastern England. - Engineering Geology, 63: 49-68.

BENN, D.I. (1990): Scottish Lateglacial moraines: debris supply, genesis and significance. - 458 S., University of St Andrews, Unpublished PhD Thesis.

BENN, D.I. (1992): The genesis and significance of ,hummocky moraine evidence from the Isle of Skye, Scotland. - Quaternary Science Reviews, 11: 781-799.

BenN, D.I. (2004a): Macrofabric. - In: Evans, D.J.A. \& BenN, D.I. (eds.): A practical guide to the study of glacial sediments: 93-114; London (Arnold).

Benn, D.I. (2004b): Clast morphology. - In: Evans, D.J.A. \& BenN, D.I (eds.): A practical guide to the study of glacial sediments: 78-92; London (Arnold)

Benn, D.I. \& Evans, D.J.A. (1993): Glaciomarine deltaic deposition and ice-marginal tectonics: the ,Loch Don Sand Moraine', Isle of Mull Scotland. - Journal of Quaternary Science, 8: 279-291.

BEnN, D.I. \& EvAns, D.J.A. (1996): The interpretation and classification of subglacially-deformed materials. - Quaternary Science Reviews, 15: 23-52.

BEnN, D.I. \& Evans, D.J.A. (1998): Glaciers and glaciation. - 734 S. London (Arnold).

BENN, D.I. \& Evans, D.J.A. (2004): Introduction and rationale. - In: Evans, D.J.A. $\&$ BENN, D.I. (eds.): A practical guide to the study of glacial sediments: 1-10; London (Arnold).

BENN, D.I. \& Evans, D.J.A. (2010): Glaciers and glaciation. Second edition. - 816 S., London (Arnold)

Benn, D.I., Evans, D.J.A., Phillips, E.R., Hiemstra, J.F., Walden, J. \& Hoey, T.B. (2004): The research project - a case study of Quaternary glacial sediments. - In: EvANs, D.J.A. \& BENN, D.I. (eds.): A practical guide to the study of glacial sediments: 209-234; London (Arnold).

BenN, D.I. \& LukAs, S. (2006): Younger Dryas glacial landsystems in North West Scotland: An assessment of modern analogues and palaeoclimatic implications. - Quaternary Science Reviews, 25: 2390-2408.

BennetT, M.R. (2001): The morphology, structural evolution and significance of push moraines. - Earth-Science Reviews, 53: 197-236.

Bennett, M.R. \& GLAsser, N.F. (2009): Glacial Geology: Ice sheets and landforms. 2nd edition. -364 S. Chichester (Wiley \& Sons).

Boulton, G.S. (1970): On the deposition of subglacial and melt-out tills at the margins of certain Svalbard glaciers. - Journal of Glaciology, 9: $231-245$.

Bradwell, T., Sigurdsson, O. \& Everest, J. (2013): Recent, very rapid retreat of a temperate glacier in SE Iceland. - Boreas, 42: 959-973.

Braun, F.J. (1987): Quartär. - In: Nordrhein-WestFalen, G.L. (ed.) Geologische Karte von Nordrhein-Westfalen 1:100 000. Erläuterungen C4306 Recklinghausen: 83-121; Krefeld (Geologisches Landesamt Nordrhein-Westfalen)

Chandler, B.M.P., Evans, D.J.A. \& Roberts, D.H. (2016): Characteristics of recessional moraines at a temperate glacier in SE Iceland: Insights into patterns, rates and drivers of glacier retreat. - Quaternary Science Reviews, 135: 171-205.

Dahm, H.-D., Deutloff, O., Klostermann, J., Knapp, G., Kühn-Velten, H., Michel, G. \& Skupin, K. (1985): Geologische Karte von Nord-
rhein-Westfalen 1:100 000. Erläuterungen C4318 Paderborn. - 60 S. Krefeld (Geologisches Landesamt).

De Saussure, H.B. (1779): Voyages dans les Alpes. 4 volumes. - (Neuchâtel) DreIMANIS, A. (1988): Tills: their genetic terminology and classification. In: GoldThwAIT, R.P. \& MATsCH, C.L. (eds.): Genetic classification of glacigenic deposits: 17-83; Rotterdam (Balkema).

Driesen, B. (1990): Erdgeschichte. - In: Driesen, B., Koch, M., Michei, G., Stehn, O. \& Wrede, V. (eds.): Geologische Karte von NordrheinWestfalen 1:100 000. Erläuterungen C 4310 Münster: 8-19; Krefeld (Geologisches Landesamt).

EHLERS, J. (1990): Gliederung der eiszeitlichen Ablagerungen in Norddeutschland. - In: LiedTKe, H. (ed.) Eiszeitforschung: 159-172; Darmstadt (Wissenschaftliche Buchgesellschaft).

EHLERs, J. (1994): Allgemeine und historische Quartärgeologie. - 358 S. Stuttgart (Enke)

EhLERS, J. (2011): Das Eiszeitalter. - 363 S. Heidelberg (Spektrum Verlag).

EvANs, D.J.A. (1989): Apron entrainment at the margins of subpolar glaciers, northwest Ellesmere Island, Canadian high arctic. - Journal of Glaciology, 35: 317-324.

Evans, D.J.A. (2009): Controlled moraines: origins, characteristics and palaeoglaciological implications. - Quaternary Science Reviews, 28: 183-208.

Evans, D.J.A. \& BenN, D.I. (2004a, eds.): A practical guide to the study of glacial sediments. - London (Arnold).

Evans, D.J.A. \& BenN, D.I. (2004b): Facies description and the logging of sedimentary exposures. - In: Evans, D.J.A. \& BENN, D.I. (eds.): A practical guide to the study of glacial sediments: $11-51$; London $(\mathrm{Ar}-$ nold).

EvANs, D.J.A. \& HARrison, S. (2014): The glacial landforms of Great Varracombe and the North Teign River. - In: Evans, D.J.A. \& HARrison, S. (eds.): The Quaternary glaciation of Dartmoor: Field Guide: 85-88; London (Quaternary Research Association).

Evans, D.J.A. \& Hiemstra, J.F. (2005): Till deposition by glacier submarginal, incremental thickening. - Earth Surface Processes and Landforms, 30: 1633-1662.

Evans, D.J.A., REA, B.R. \& BENN, D.I. (1998): Subglacial deformation and bedrock plucking in areas of hard bedrock. - Glacial Geology and Geomorphology, (rp04/1998 - http://ggg.qub.ac.uk/ggg/papers/ full/1998/rp041998/rp04.html).

Evans, D.J.A., Phillips, E.R., Hiemstra, J.F. \& Auton, C.A. (2006): Subglacial till: Formation, sedimentary characteristics and classification - Earth-Science Reviews, 78: 115-176.

Evans, D.J.A., Rother, H., Hyatt, O. \& Shulmeister, J. (2013): The glacial sedimentology and geomorphic evolution of an outwash head/ moraine-dammed lake, South Island, New Zealand. - Sedimentary Geology, 284: 45-75.

Eyles, N., Eyles, C.H. ¿ MiAll, A.D. (1983): Lithofacies types and vertical profile models. An alternative approach to the description and environmental interpretation of glacial diamict and diamictite sequences. - Sedimentology, 30: 393-410.

FLINT, R.F. (1971): Glacial and Quaternary geology. - 892 S. New York (Wiley \& Sons)

Frakes, L.A. (1978): Diamictite. - In: FAIrbridge, R.W. ¿ Bourgeois, J (eds.): The encyclopedia of sedimentology: 262-263; Stroudsburg (Dowden, Hutchinson and Ross).

Geikie, A. (1863): On the glacial drift of Scotland. - Transactions of the Geological Society of Glasgow, 1: 1-190.

GlatthaAR, D. (1998): Die Strandterrassen am Südufer des Liefdefjordes, Spitzbergen. - Bochumer Geographische Arbeiten, Sonderreihe, 13: $10-13$.

GlatthaAR, D. \& LiedtKe, H. (1981): Die Entwicklung des Ruhrtals und glazialmorphologische Probleme im Bochumer Raum. - Bochumer Geographische Arbeiten, 40: 99-102.

GoldThwAIT, R.P. (1971): Introduction to till, today. - In: GoldTHwAIT, R.P. (ed.) Till - a symposium: 3-26; Ohio (Ohio State Univeristy Press)

Griffiths, J. \& MARTIN, C.J. (2016): Engineering Geology of glacial and periglacial sediments. - In: Griffiths, J. \& MARTIN, C. (eds.): Engineering Geology of glacial and periglacial sediments. - Geological Society of London, London, in press.

GRIPP, K. (1975): Hochsander - Satzmoräne - Endmoränenvertreter. Zeitschrift für Geomorphologie, 19: 490-496.

GRUBE, F. (1990): Zur Morphogenese und Sedimentation im quartären Ver eisungsgebiet Nordwestdeutschlands. - In: LiedtKe, H. (ed.) Eiszeitforschung: 220-230; Darmstadt (Wissenschaftliche Buchgesellschaft).

Haldorsen, S., \& SHaw, J. (1982): The problem of recognizing melt out till. - Boreas 11: 261-277. 
Hantke, R. \& Wagner, G. (2004): Ältere Berner Schotter und eiszeitliche Mittelmoränen. - Mitteilungen der Naturforschenden Gesellschaft in Bern, 61: 101-125.

Herget, J. (1997): Die Flußentwicklung des Lippetals. - Bochumer Geographische Arbeiten, 62: 1-132.

Herget, J. (1998): Temporäre Entwässerungsbahnen am Südrand der Westfälischen Tieflandsbucht - ein Szenario. - Bochumer Geographische Arbeiten Sonderreihe, 13: 23-30.

Нiсоск, S.R. (1990): Genetic till prism. - Geology, 18: 517-519.

Hiemstra, J.F., Matthews, J.A., Evans, D.J. \& Owen, G. (2015): Sediment fingerprinting and the mode of formation of singular and composite annual moraine ridges at two glacier margins, Jotunheimen, southern Norway. - The Holocene, 25: 1772-1785.

Hornby, A.S. et al. (ed.) (1989): Oxford Advanced Learner's Dictionary. 1579 S. Oxford (Oxford University Press).

Kaiser, K., Schoknecht, T., Janke, W., Kloss, K. \& Prehn, B. (2002): Geomorphologische, palynologische und archäologische Beiträge zur holozänen Landschaftsgeschichte im Müritzgebiet (Mecklenburg-Vorpommern). - Eiszeitalter und Gegenwart, 51: 15-32.

KAMP, U. \& HASERODT, K. (2002): Quartäre Vergletscherungen in Hindukusch, Karakorum und West-Himalaya, Pakistan - Ein Überblick. Eiszeitalter und Gegenwart, 51: 93-113.

Karte, J. (1998): Zum Problem der Konfluenz von Rocky Mountains-Vorlandgletschern und laurentischem Inlandeis in West-Alberta, Kanada. - Bochumer Geographische Arbeiten, Sonderreihe, 13: 31-36.

KIRKBride, M.P. \& WINkLER, S. (2012): Correlation of Late Quaternary moraines: impact of climate variability, glacier response, and chronological resolution. - Quaternary Science Reviews, 46: 1-29.

KRIENKE, K. (2003): Südostrügen im Hochglazial - Lithostratigraphie, strukturgeologische und landschaftsgenetische Studien zur jüngsten Vergletscherung im Küstenraum Vorpommers (NE-Deutschland). Greifswalder Geowissenschaftliche Beiträge, 12: 1-147.

KRIENKE, K. (2004): Das Geschiebeinventar der weichselhochglazialen Tills von Südostrügen - Hilfsmittel zur Lithostratigraphie sowie zur Rekonstruktion von Ablagerungsbedingungen und glazialer Dynamik. - Archiv für Geschiebekunde, 3: 701-710.

KRÜGER, J. (1994): Glacial processes, sediments, landforms, and stratigraphy in the terminus region of Myrdalsjökull, Iceland. - Folia Geographica Danica, 21: 1-233.

KRÜGER, J. (1995): Origin, chronology and climatological significance of annual-moraine ridges at Myrdalsjökull, Iceland. - The Holocene, 5 420-427.

KRÜGER, J. ひ ABER, J.S. (1999): Formation of supraglacial sediment accumulations on Kötlujökull, Iceland. - Journal of Glaciology, 45: 400-402.

KRÜGER, J. \& KJÆR, K.H. (1999): A data chart for field description and genetic interpretation of glacial diamicts and associated sediments with examples from Greenland, Iceland, and Denmark. - Boreas, 28: 386-402.

Kuhle, M. (2002): Die eiszeitliche Vergletscherung des Karakorum - Rekonstruktion der maximalen Gletscherausdehnung und Eismächtigkeiten. - Terra Nostra, 2002/6: 178-184.

Kühn, P., Janetzko, P. ひ Schröder, D. (2002): Zur Mikromorphologie und Genese lessivierter Böden im Jungmoränengebiet SchleswigHolsteins und Mecklenburg-Vorpommerns. - Eiszeitalter und Gegenwart, 51: 74-92.

Lawson, D.E. (1981): Distinguishing characteristics of diamictons at the margin of the Matanuska glacier, Alaska. - Annals of Glaciology, 2: 78-84.

LAwson, D.E. (1982): Mobilization, movement and deposition of active subaerial sediment flows, Matanuska Glacier, Alaska. - Journal of Geology, 90: 279-300.

LAwson, D.E. (1988): Glacigenic resedimentation: Classification concepts and application to mass-movement processes and deposits. - In: GoldThwAIT, R.P. \& MATSCH, C.L. (eds.): Genetic classification of glacigenic deposits: 147-169; Rotterdam (Balkema).

LiedTKe, H. (1981): Die nordischen Vereisungen in Mitteleuropa. 2. Auflage. - Forschungen zur deutschen Landeskunde, 204: 1-307.

Liedtke, H. (1990): Stand und Aufgabe der Eiszeitforschung. - In: LiedTKE, H. (ed.) Eiszeitforschung: 40-54; Darmstadt (Wissenschaftliche Buchgesellschaft).

Louis, H. \& Fischer, K. (1979): Allgemeine Geomorphologie. - 814 S., Berlin (de Gruyter)

LovelL, H. (2014): On the ice-sediment-landform associations of surging glaciers on Svalbard. - 295 S., Queen Mary University of London, Unpublished $\mathrm{PhD}$ thesis.
Lovell, H., Fleming, E.J., Benn, D.I., Hubbard, B., Lukas, S., Rea, B.R., Noormets, R. \& FLINK, A.E. (2015): Debris entrainment and landform genesis during tidewater glacier surges. - Journal of Geophysical Research: Earth Surface: n/a-n/a.

LowE, D.R. (1975): Water escape structures in coarse-grained sediments. Sedimentology, 22: 157-204.

Ludwig, A.O. (2005): Zur Interpretation des Kliffanschnitts östlich von Glowe / Insel Rügen (Ostsee). - Zeitschrift für geologische Wissenschaften (Berlin) 33: 263-272.

LukAs, S. (2003): The moraines around the Pass of Drumochter. - Scottish Geographical Journal, 119: 383-393.

LuKas, S. (2004): Moräne oder Till? Ein Vorschlag zur Beschreibung, Interpretation und Benennung glazigener Sedimente. - Zeitschrift für Gletscherkunde und Glazialgeologie, 39: 141-159.

LuKAS, S. (2005): A test of the englacial thrusting hypothesis of ,hummocky' moraine formation - case studies from the north-west Highlands, Scotland. - Boreas, 34: 287-307.

LuKAs, S. (2007): Englacial thrusting and (hummocky) moraine formation: a reply to comments by Graham et al. (2007). - Boreas, 36: 108-113.

Lukas, S. (2011): Ice-cored moraines. - In: Singh, V., Singh, P. \& HARITASHYA, U.K. (eds.): Encyclopedia of Snow, Ice and Glaciers: 616-619; Heidelberg (Springer).

LuKAs, S. (2012): Processes of annual moraine formation at a temperate alpine valley glacier: insights into glacier dynamics and climatic controls. - Boreas, 41: 463-480.

LuKAs, S. \& SAss, O. (2011): The formation of Alpine lateral moraines inferred from sedimentology and radar reflection patterns - a case study from Gornergletscher, Switzerland. - Geological Society of London Special Publications, 354: 77-92.

Lukas, S., Nicholson, L.I., Ross, F.H. \& Humlum, O. (2005): Formation, meltout processes and landscape alteration of high-arctic ice-cored moraines - examples from Nordenskiöld Land, central Spitsbergen. Polar Geography, 29: 157-187.

Lukas, S., Nicholson, L.I. \& Humlum, O. (2007): Comment on Lønne and Lyså 2005: Deglaciation dynamics following the Little Ice Age on Svalbard: Implications for shaping of landscapes at high latitudes. Geomorphology 72, 300-319. - Geomorphology, 84: 145-149.

Lukas, S., Graf, A., Coray, S. \& Schlüchter, C. (2012): Genesis, stability and preservation potential of large lateral moraines of Alpine valley glaciers - towards a unifying theory based on Findelengletscher, Switzerland. - Quaternary Science Reviews, 38: 27-48.

Lukas, S., Benn, D.I., Boston, C.M., Brook, M.S., Coray, S., Evans, D.J.A., Graf, A., Kellerer-Pirklbauer-Eulenstein, A., Kirkbride, M.P., Krabbendam, M., Lovell, H., Machiedo, M., Mills, S.C., Nye, K., Reinardy, B.T.I., Ross, F.H. \& Signer, M. (2013): Clast shape analysis and clast transport paths in glacial environments: A critical review of methods and the role of lithology. - Earth-Science Reviews, 121: 96-116.

LuNDQVisT, J. (1989): Till and glacial landforms in a dry, polar region. Zeitschrift für Geomorphologie, 33: 27-41.

Lüthgens, C. \& Böse, M. (2012): From Morphostratigraphy to Geochronology - on the dating of ice marginal positions. Quaternary Science Reviews, 44: 26-36.

Matthews, J.A., Mccarroll, D. \& Shakesby, R.A. (1995): Contemporary terminal-moraine ridge formation at a temperate glacier: Styggedalsbreen, Jotunheimen, southern Norway. - Boreas, 24: 129-139.

OkadA, H. \& Kenyon-Smith, A. (2009): The birth of sedimentology: Henry Clifton Sorby and Johannes Walther. - Geology Today, 25: 211-218.

Owen, L.A. (1994): Glacial and non-glacial diamictons in the Karakorum Mountains and western Himalayas. - In: WARren, W.P. \& CROOT, D.G. (eds.): Formation and deformation of glacial deposits: 9-28; Rotterdam (Balkema)

PAul, M.A. \& Eyles, N. (1990): Constraints on the preservation of diamict facies (melt-out tills) at the margins of stagnant glaciers. - Quaternary Science Reviews, 9: 51-69.

Phillips, E. (2006): Micromorphology of a debris flow deposit: evidence of basal shearing, hydrofracturing, liquefaction and rotational deformation during emplacement. - Quaternary Science Reviews, 25: $720-738$.

Phillips, E., Lee, J.R., Riding, J.B., Kendall, R. \& Hughes, L. (2013): Periglacial disruption and subsequent glacitectonic deformation of bedrock: an example from Anglesey, North Wales, UK. - Proceedings of the Geologists' Association, 124: 802-817.

Pindur, P. \& Heuberger, H. (2008): Zur holozänen Gletschergeschichte im Zemmgrund in den Zillertaler Alpen, Tirol/Österreich (Ostalpen). - Zeitschrift für Gletscherkunde und Glazialgeologie, 42: 21-89. 
Piotrowski, J.A. (1992): Was ist ein Till? Faziesstudien an glazialen Sedimenten. - Die Geowissenschaften, 10: 100-108.

Piotrowski, J.A., Mickelson, D.M., Tulaczyk, S., Krzyszkowski, D. \& JUNGE, F.W. (2001): Were subglacial deforming beds beneath past ice sheets really widespread? - Quaternary International, 86: 139-150.

Preusser, F., Deggering, D., Fuchs, M., Hilgers, A., Kadereit, A., KlaSEN, N., KrbetscheK, M., RichteR, D. \& SPENCER, J.Q.G. (2008): Luminescence dating: Basics, methods and applications. - Eiszeitalter und Gegenwart, 57: 95-149.

READING, H.G. (1996, ed.): Sedimentary environments: processes, facies and stratigraphy. - 688 S., Oxford (Blackwell).

REINARDY, B.T.I. ¿ LUKAS, S. (2009): A comparison of the sedimentary signature of ice-contact sedimentation and deformation at macro- and micro-scale: a case study from NW Scotland. - Sedimentary Geology, 221: 87-98.

ReinARDY, B.T.I., LeIghton, I. \& MARX, P.J. (2013): Glacier thermal regime linked to processes of annual moraine formation at Midtdalsbreen, southern Norway. - Boreas, 42: 896-911.

Rother, H., SHulmeister, J. $\mho$ Rieser, U. (2010): Stratigraphy, optical dating chronology (IRSL) and depositional model of pre-LGM glacia deposits in the Hope Valley, New Zealand. - Quaternary Science Reviews, 29: 576-592.

SchlǗhter, C. (1999): Sedimente des Gletschers (Teil II). - Bulletin für Angewandte Geologie, 4: 67-75.

Sharp, M. (1984): Annual moraine ridges at Skàlafellsjökull, south-east Iceland. - Journal of Glaciology, 30: 82-93.

Sharpe, D.R. (1988): Late glacial landforms of Wollaston Peninsula, Victoria Island, NWT: product of ice-marginal retreat, surge, and mass stagnation. - Canadian Journal of Earth Sciences 25: 262-279.

STоw, D.A.V. (2008): Sedimentgesteine im Gelände. - Springer Spektrum, $315 \mathrm{~S}$

TulaczyK, S., Kamb, B. ש Engelhardt, H. (2000): Basal mechanics of Ice Stream B. I. Till mechanics. - Journal of Geophysical Research, 105 463-481.
TulaczyK, S., SCHERER, R. ¿ Clark, C.D. (2001): A ploughing model for the origin of weak tills beneath ice streams: a qualitative treatment. Quaternary International, 86: 59-70.

van Der MeER, J.J.M., KJÆR, K.H. \& KRÜGER, J. (2001): Subglacial waterescape structures and till structures, Sléttjökull, Iceland. - Journal of Quaternary Science, 14: 191-205.

van Der Meer, J.J.M., Menzies, J. \& Rose, J. (2003): Subglacial till: the deforming glacier bed. - Quaternary Science Reviews, 22: 1659-1685.

van Der Wateren, F.M. (1995): Structural geology and sedimentology of push moraines - processes of soft sediment deformation in a glacial environment and the distribution of glaciotectonic styles. - Mededelingen Rijks Geologische Dienst, 54: 1-168.

van Der Wateren, F.M., Kluiving, S.J. ¿ Bartek, L.R. (2000): Kinematic indicators of subglacial shearing. - In: Maltman, A.J., Hubbard, B. \& HAMBREY, M.J. (eds.): Deformation of glacial materials. Geological Society of London Special Publication 176: 259-278).

van HusEN, D. (1990): Verbreitung, Ursachen und Füllung glazial übertiefter Talabschnitte an Beispielen in den Ostalpen. - In: LiedTKe, H. (ed.) Eiszeitforschung: 203-219; Darmstadt (Wissenschaftliche Buchgesellschaft).

von BöHMERSheim, A.B.E. (1901): Geschichte der Moränenkunde. - Abhandlungen der König-Kaiserlichen Gesellschaft in Wien, 3: 1-334.

WinkLER, S. (2009): Gletscher und ihre Landschaften - eine illustrierte Einführung. - 183 S. Darmstadt (Wissenschaftliche Buchgesellschaft)

WinkLER, S. \& HAGEDORN, H. (1999): Lateralmoränen - Morphologie, Genese und Beziehung zu Gletscherstandsschwankungen (Beispiele aus Ostalpen und West-/Zentralnorwegen). - Zeitschrift für Geomorphologie, Supplement Band, 113: 69-84.

WinKLER, S. \& NesJe, A. (1999): Moraine formation at an advancing temperate glacier: Brigsdalsbreen, western Norway. - Geografiska Annaler, 81A: $17-30$

Zepp, H. (2002): Grundriß allgemeine Geographie: Geomorphologie. 1. Auflage. - 354 S. Paderborn (Schöningh) 